\title{
Icehouse-greenhouse variations in marine denitrification
}

\author{
T. J. Algeo ${ }^{1}$, P. A. Meyers ${ }^{2}$, R. S. Robinson ${ }^{3}$, H. Rowe ${ }^{4}$, and G. Q. Jiang ${ }^{5}$ \\ ${ }^{1}$ Department of Geology, University of Cincinnati, Cincinnati, OH 45221-0013, USA \\ ${ }^{2}$ Department of Geological Sciences, University of Michigan, Ann Arbor, MI 48109-1063, USA \\ ${ }^{3}$ Graduate School of Oceanography, University of Rhode Island, Narragansett, RI 02882, USA \\ ${ }^{4}$ Department of Earth and Environmental Sciences, University of Texas at Arlington, Arlington, TX 76019, USA \\ ${ }^{5}$ Department of Geoscience, University of Nevada Las Vegas, Las Vegas, Nevada, USA \\ Correspondence to: T. J. Algeo (thomas.algeo@uc.edu)
}

Received: 12 August 2013 - Published in Biogeosciences Discuss.: 6 September 2013

Revised: 18 January 2014 - Accepted: 25 January 2014 - Published: 27 February 2014

\begin{abstract}
Long-term secular variation in the isotopic composition of seawater fixed nitrogen $(\mathrm{N})$ is poorly known. Here, we document variation in the $\mathrm{N}$-isotopic composition of marine sediments $\left(\delta^{15} \mathrm{~N}_{\mathrm{sed}}\right)$ since $660 \mathrm{Ma}$ (million years ago) in order to understand major changes in the marine $\mathrm{N}$ cycle through time and their relationship to first-order climate variation. During the Phanerozoic, greenhouse climate modes were characterized by low $\delta^{15} \mathrm{~N}_{\text {sed }}(\sim-2$ to $+2 \%$ ) and icehouse climate modes by high $\delta^{15} \mathrm{~N}_{\text {sed }}(\sim+4$ to $+8 \%$ ). Shifts toward higher $\delta^{15} \mathrm{~N}_{\text {sed }}$ occurred rapidly during the early stages of icehouse modes, prior to the development of major continental glaciation, suggesting a potentially important role for the marine $\mathrm{N}$ cycle in long-term climate change. Reservoir box modeling of the marine $\mathrm{N}$ cycle demonstrates that secular variation in $\delta^{15} \mathrm{~N}_{\text {sed }}$ was likely due to changes in the dominant locus of denitrification, with a shift in favor of sedimentary denitrification during greenhouse modes owing to higher eustatic (global sea-level) elevations and greater on-shelf burial of organic matter, and a shift in favor of water-column denitrification during icehouse modes owing to lower eustatic elevations, enhanced organic carbon sinking fluxes, and expanded oceanic oxygen-minimum zones. The results of this study provide new insights into operation of the marine $\mathrm{N}$ cycle, its relationship to the global carbon cycle, and its potential role in modulating climate change at multimillion-year timescales.
\end{abstract}

\section{Introduction}

Nitrogen $(\mathrm{N})$ plays a key role in marine productivity and organic carbon fluxes and is thus a potentially major influence on the global climate system (Gruber and Galloway, 2008). Variation in marine sediment $\mathrm{N}$-isotopic compositions during the Quaternary (2.6 Ma to the present) has been linked to changes in organic carbon burial and oceanic denitrification rates during Pleistocene glacial-interglacial cycles (François et al., 1992; Altabet et al., 1995; Ganeshram et al., 1995; Haug et al., 1998; Naqvi et al., 1998; Broecker and Henderson, 1998; Suthhof et al., 2001; Liu et al., 2005, 2008). At this timescale (i.e., $\sim 10^{5} \mathrm{yr}$ ), the marine $\mathrm{N}$ cycle is thought to act mainly as a positive climate feedback, but negative feedbacks involving the influence of both $\mathrm{N}$ fixation and denitrification on oceanic fixed-N inventories have been proposed as well (Deutsch et al., 2004). Although preQuaternary $\delta^{15} \mathrm{~N}_{\text {sed }}$ variation has been reported, including highly ${ }^{15} \mathrm{~N}$-depleted ( -4 to $0 \%$ ) Jurassic-Cretaceous units (Rau et al., 1987; Jenkyns et al., 2001; Junium and Arthur, $2007)$ and highly ${ }^{15} \mathrm{~N}$-enriched $(+6$ to $+14 \%$ ) Carboniferous units (Algeo et al., 2008), the Phanerozoic record of marine $\mathrm{N}$-isotopic variation and its relationship to long-term (i.e., multimillion-year) climate change have not been systemically investigated to date (Algeo and Meyers, 2009). Additional study of the marine $\mathrm{N}$ cycle is needed to better understand its relationship to organic carbon burial and long-term climate change and to more accurately parameterize $\mathrm{N}$ fluxes in general circulation models. In this study, we document variation in $\delta^{15} \mathrm{~N}_{\text {sed }}$ from 660 Ma to the present, demonstrating a strong relationship to first-order climate cycles, with 


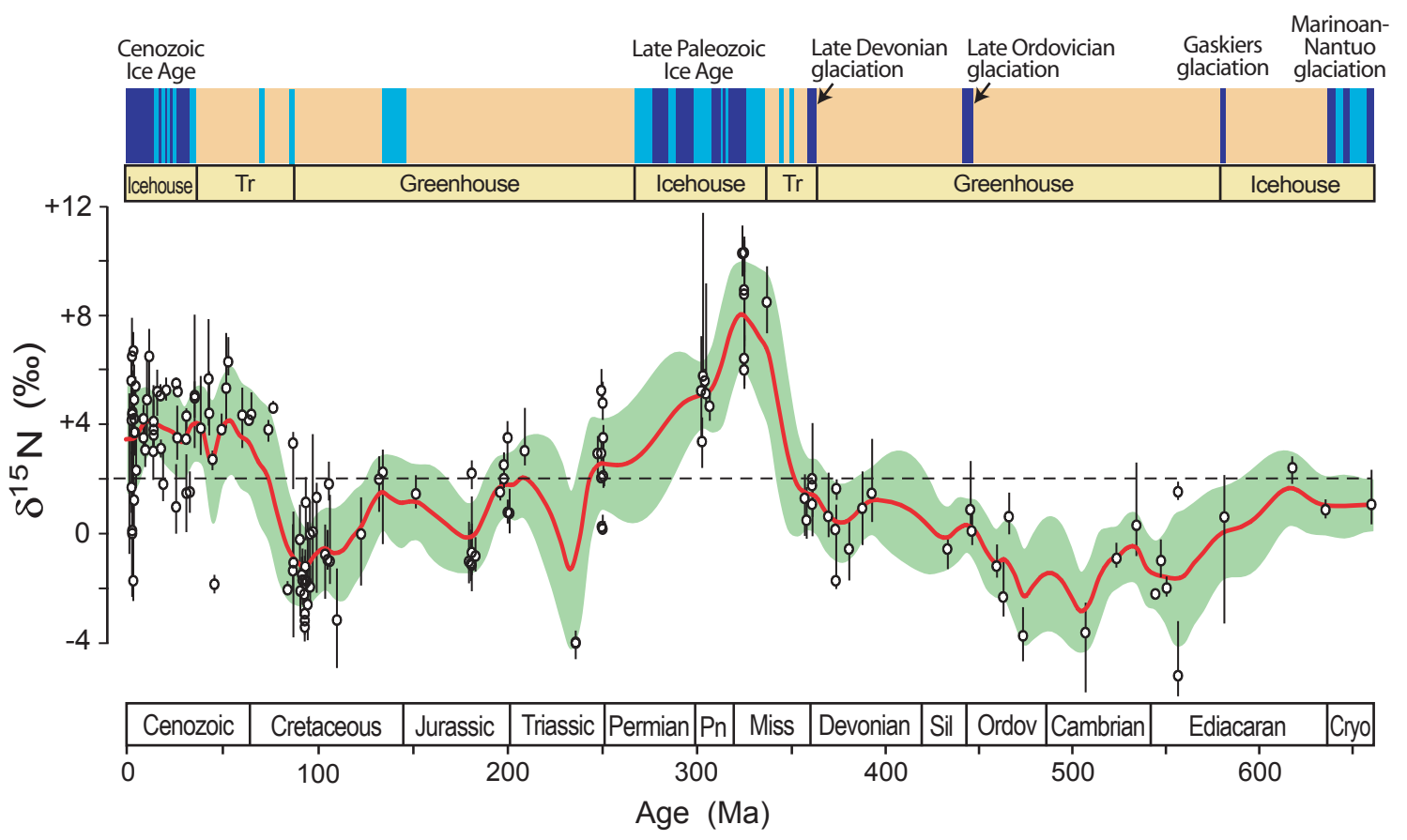

Fig. 1. Long-term secular variation in marine sediment $\delta^{15} \mathrm{~N}$, based on 153 study units of Neoproterozoic and Phanerozoic age. This data set yields a mean $\delta^{15} \mathrm{~N}_{\text {sed }}$ of $+2.0 \pm 0.3 \%$ (mean \pm 1 standard error of the mean; dashed line). For each unit, the distribution of $\delta^{15} \mathrm{~N}$ values is represented by the median (open circle) and the 16th-to-84th percentile range (vertical line) (see Table 1). The mean long-term trend is given by a LOWESS curve (red line) and uncertainty envelope $( \pm 1 \sigma$; green field). The LOWESS curve, which varies over a $\sim 10 \%$ o range, accounts for $74 \%$ of total variance in the $\delta^{15} \mathrm{~N}_{\text {sed }}$ data set. At top, epochs of moderate (light blue) and heavy (dark blue) continental glaciation are from Montañez et al. (2011); the ages of all marine sedimentary units and climate events have been adjusted to the timescale of Gradstein et al. (2012). $\operatorname{Tr}=$ transitional interval.

lower $\delta^{15} \mathrm{~N}$ during greenhouse intervals and higher $\delta^{15} \mathrm{~N}$ during icehouse intervals. This pattern suggests that long-term variation in the marine $\mathrm{N}$ cycle is controlled by first-order tectonic cycles, and that it is linked to (is a possibly a driver of) long-term climate change.

\section{Methods}

This study is based on the N-isotope distributions of 153 marine units ranging in age from the Neoproterozoic $(660 \mathrm{Ma})$ to the early Quaternary ( $\sim 2 \mathrm{Ma}$ ) (Fig. 1). Among these units are 35 that were analyzed specifically for this study (see isotopic methods, Appendix A), 33 that were taken from our own earlier research publications, and 85 that were taken from other published reports. For each study unit, we determined the median (50th percentile), standard deviation range (16th and 84th percentiles), and full range (minimum and maximum values) of its $\delta^{15} \mathrm{~N}$ distribution (Table 1). We also report organic $\delta^{13} \mathrm{C}$ distributions as well as means for \% TOC (total organic carbon), $\% \mathrm{~N}$, and molar $\mathrm{C}_{\text {org }}: \mathrm{N}$ ratios, where available (Table 1). The ages of all units were adjusted to the 2012 geologic timescale (Gradstein et al., 2012). A LOWESS (LOcally WEighted Scatterplot Smoothing) curve was calculated for the entire data set per the methods of Appendix B.

\section{Results}

Our $\delta^{15} \mathrm{~N}_{\text {sed }}$ data set exhibits a mean plus/minus one standard deviation of $+2.0 \pm 3.1 \%$ with a range of -5.2 to $+10.4 \%$ o (Table $1 ; n=153) . \delta^{15} \mathrm{~N}_{\text {sed }}$ values are mostly intermediate ( 0 to $+3 \%$ ) during the late Cryogenian to early Ediacaran, low ( -5 to $0 \%$ ) during the late Ediacaran to mid-Ordovician, intermediate during the Late Ordovician to Early Mississippian, high $(+3$ to $+10 \%$ ) during the Late Mississippian to Pennsylvanian, intermediate during the Triassic to Early Cretaceous, low during the mid-Cretaceous, and intermediate to high during the Late Cretaceous to Recent (Fig. 1). The modeled LOWESS curve for the Phanerozoic exhibits a minimum of $-2.8 \%$ in the Cambrian and a maximum of $+8.0 \%$ o in the Mississippian. The uncertainty attached to this mean trend varies from \pm 0.9 to $2.9 \%$ o through the Phanerozoic but is mostly $< \pm 2 \%$ (based on plus/minus one standard deviation). The most abrupt changes in $\delta^{15} \mathrm{~N}_{\text {sed }}$ are associated with a $\sim 6 \%$ o rise during the mid-Mississippian and a $\sim 5 \%$ o rise during the Late Cretaceous. The Phanerozoic $\delta^{15} \mathrm{~N}_{\text {sed }}$ curve shows a strong relationship to first-order climate cycles, with low values during the greenhouse climate modes of the mid-Paleozoic and mid-Mesozoic and high values during the icehouse climate modes of the Late Paleozoic and Cenozoic (Fig. 1). 
Table 1. N-isotope and correlative data for 153 marine units from 660 Ma to Recent.

\begin{tabular}{|c|c|c|c|c|c|c|c|c|c|c|}
\hline Record & Source & Location & Formation & Setting & Period & Series & $\begin{array}{l}\text { Start } \\
(\mathrm{Ma})\end{array}$ & $\begin{array}{l}\text { Age } \\
\text { End } \\
\text { (Ma) }\end{array}$ & $\begin{array}{l}\text { Mid } \\
(\mathrm{Ma})\end{array}$ & $\begin{array}{l}\text { Sample } \\
n\end{array}$ \\
\hline 1 & Meyers (unpubl.) & California margin (ODP 1014) & unnamed & upwelling & $\mathrm{Ng}$ & Pleistocene & 1.93 & 2.26 & 2.10 & 84 \\
\hline 2 & Robinson and Meyers (2002) & $\begin{array}{l}\text { Namibian Shelf (ODP 1082- } \\
\text { 1084) }\end{array}$ & unnamed & upwelling & $\mathrm{Ng}$ & Pleistocene & 1.95 & 2.45 & 2.20 & 195 \\
\hline 3 & Macko and Pereira (1990) & Antarctic margin (ODP 693) & unnamed & oceanic & $\mathrm{Ng}$ & Pleistocene & 2.60 & 1.80 & 2.20 & 60 \\
\hline 4 & Muzuka et al. (1991) & Oman margin & unnamed & upwelling & $\mathrm{Ng}$ & Pleistocene & 3.00 & 1.80 & 2.40 & 254 \\
\hline 5 & Arnaboldi and Meyers (2006) & Mediterranean (974C) & unnamed & oceanic-med & $\mathrm{Ng}$ & Pleistocene & 3.00 & 2.00 & 2.50 & 97 \\
\hline 6 & Arnaboldi and Meyers (2006) & Mediterranean (969D) & unnamed & oceanic-med & $\mathrm{Ng}$ & Pleistocene & 3.00 & 2.00 & 2.50 & 93 \\
\hline 7 & Arnaboldi and Meyers (2006) & Mediterranean (967B) & unnamed & oceanic-med & $\mathrm{Ng}$ & Pleistocene & 3.00 & 2.00 & 2.50 & 50 \\
\hline 8 & $\mathrm{Li}$ and Bebout (2006) & Costa Rica margin (ODP 170) & unnamed & oceanic & $\mathrm{Ng}$ & Plio-Pleistocene & 5.30 & 0.00 & 2.65 & 52 \\
\hline 9 & Pedersen (unpubl.) & Mediterranean (969B) & unnamed & oceanic-med & $\mathrm{Ng}$ & Pliocene & 3.06 & 3.05 & 3.06 & 24 \\
\hline 10 & Liu et al. (2008) & E Trop Pacific (ODP 1012) & unnamed & upwelling & $\mathrm{Ng}$ & Pliocene & 4.10 & 2.10 & 3.10 & 721 \\
\hline 11 & Struck et al. (2001) & Mediterranean & unnamed & oceanic-med & $\mathrm{Ng}$ & Pliocene & 5.00 & 2.00 & 3.50 & 49 \\
\hline 12 & Li and Bebout (2006) & Costa Rica margin (ODP 205) & unnamed & oceanic & $\mathrm{Ng}$ & Pliocene & 5.30 & 1.80 & 3.55 & 13 \\
\hline 13 & Macko and Pereira (1990) & Antarctic margin (ODP 694) & unnamed & oceanic & $\mathrm{Ng}$ & Pliocene & 5.30 & 1.80 & 3.55 & 82 \\
\hline 14 & Macko and Pereira (1990) & Antarctic margin (ODP 690) & unnamed & oceanic & $\mathrm{Ng}$ & Pliocene & 5.30 & 1.80 & 3.55 & 40 \\
\hline 15 & Macko and Pereira (1990) & Antarctic margin (ODP 693) & unnamed & oceanic & $\mathrm{Ng}$ & IPliocene & 5.30 & 2.60 & 3.95 & 53 \\
\hline 16 & Sadofsky and Bebout (2004) & western Pacific (ODP 1149) & unnamed & oceanic & $\mathrm{Ng}$ & uMio-Pliocene & 6.50 & 2.00 & 4.25 & 11 \\
\hline 17 & Macko and Pereira (1990) & Antarctic margin (ODP 689) & unnamed & oceanic & $\mathrm{Ng}$ & Pliocene & 5.30 & 4.00 & 4.65 & 29 \\
\hline 18 & Macko and Pereira (1990) & Antarctic margin (ODP 693) & unnamed & oceanic & $\mathrm{Ng}$ & uMiocene & 11.61 & 5.30 & 8.46 & 49 \\
\hline 19 & Macko and Pereira (1990) & Antarctic margin (ODP 694) & unnamed & oceanic & $\mathrm{Ng}$ & uMiocene & 11.61 & 5.30 & 8.46 & 71 \\
\hline 20 & Macko and Pereira (1990) & Antarctic margin (ODP 689) & unnamed & oceanic & $\mathrm{Ng}$ & uMiocene & 11.61 & 7.20 & 9.41 & 22 \\
\hline 21 & Macko and Pereira (1990) & Antarctic margin (ODP 690) & unnamed & oceanic & $\mathrm{Ng}$ & uMiocene & 11.61 & 9.01 & 10.31 & 15 \\
\hline 22 & Calvert (2000) & California & Monterey & upwelling & $\mathrm{Ng}$ & Miocene & 13.51 & 9.51 & 11.51 & 23 \\
\hline 23 & Hudson et al. (2008); Rowe (unpubl.) & Azerbaijan & post-Maikop & oceanic-med & $\mathrm{Ng}$ & mMiocene & 14.82 & 12.51 & 13.67 & 17 \\
\hline 24 & Macko and Pereira (1990) & Antarctic margin (ODP 689) & unnamed & oceanic & $\mathrm{Ng}$ & mMiocene & 16.02 & 11.61 & 13.82 & 19 \\
\hline 25 & Macko and Pereira (1990) & Antarctic margin (ODP 694) & unnamed & oceanic & $\mathrm{Ng}$ & mMiocene & 16.02 & 11.61 & 13.82 & 86 \\
\hline 26 & Macko and Pereira (1990) & Antarctic margin (ODP 690) & unnamed & oceanic & $\mathrm{Ng}$ & mMiocene & 16.02 & 11.61 & 13.82 & 13 \\
\hline 27 & Macko and Pereira (1990) & Antarctic margin (ODP 693) & unnamed & oceanic & $\mathrm{Ng}$ & mMiocene & 18.02 & 13.61 & 15.82 & 25 \\
\hline 28 & Macko and Pereira (1990) & Antarctic margin (ODP 690) & unnamed & oceanic & $\mathrm{Ng}$ & lMiocene & 18.42 & 16.52 & 17.47 & 4 \\
\hline 29 & Macko and Pereira (1990) & Antarctic margin (ODP 689) & unnamed & oceanic & $\mathrm{Ng}$ & IMiocene & 19.42 & 16.02 & 17.72 & 9 \\
\hline 30 & Hudson et al. (2008); Rowe (unpubl.) & Azerbaijan & Maikop Series & oceanic-med & $\mathrm{Ng}$ & IMiocene & 23.03 & 14.82 & 18.93 & 22 \\
\hline 31 & Macko and Pereira (1990) & Antarctic margin (ODP 693) & unnamed & oceanic & $\mathrm{Ng}$ & IMiocene & 23.03 & 18.02 & 20.53 & 10 \\
\hline 32 & Hudson et al. (2008); Rowe (unpubl.) & Azerbaijan & Maikop Series & oceanic-med & $\mathrm{Pg}$ & uOligocene & 28.37 & 23.03 & 25.70 & 62 \\
\hline 33 & Macko and Pereira (1990) & Antarctic margin (ODP 693) & unnamed & oceanic & $\mathrm{Pg}$ & uOligocene & 28.47 & 23.03 & 25.75 & 11 \\
\hline 34 & Macko and Pereira (1990) & Antarctic margin (ODP 689) & unnamed & oceanic & $\mathrm{Pg}$ & uOligocene & 28.37 & 24.22 & 26.30 & 12 \\
\hline 35 & Macko and Pereira (1990) & Antarctic margin (ODP 690) & unnamed & oceanic & $\mathrm{Pg}$ & uOligocene & 28.17 & 25.01 & 26.59 & 11 \\
\hline 36 & Macko and Pereira (1990) & Antarctic margin (ODP 693) & unnamed & oceanic & Pg & Oligocene & 31.92 & 29.95 & 30.94 & 24 \\
\hline 37 & Hudson et al. (2008); Rowe (unpubl.) & Azerbaijan & Maikop Series & oceanic-med & $\mathrm{Pg}$ & lOligocene & 33.90 & 28.37 & 31.14 & 53 \\
\hline 38 & Macko and Pereira (1990) & Antarctic margin (ODP 689) & unnamed & oceanic & $\mathrm{Pg}$ & IOligocene & 33.90 & 28.37 & 31.14 & 11 \\
\hline 39 & Schulz et al. (2002) & Austria & Schoeneck & epeiric & $\mathrm{Pg}$ & Oligocene & 34.41 & 31.43 & 32.92 & 50 \\
\hline 40 & Hudson et al. (2008); Rowe (unpubl.) & Azerbaijan & Koun Fm & oceanic-med & $\mathrm{Pg}$ & uEocene & 37.14 & 33.90 & 35.52 & 75 \\
\hline 41 & Macko and Pereira (1990) & Antarctic margin (ODP 689) & unnamed & oceanic & $\mathrm{Pg}$ & uEocene & 37.14 & 33.90 & 35.52 & 16 \\
\hline 42 & Macko and Pereira (1990) & Antarctic margin (ODP 689) & unnamed & oceanic & $\mathrm{Pg}$ & mEocene2 & 40.39 & 37.14 & 38.77 & 8 \\
\hline 43 & Hudson et al. (2008); Rowe (unpubl.) & Azerbaijan & Koun Fm & oceanic-med & $\mathrm{Pg}$ & mEocene & 48.70 & 37.14 & 42.92 & 52 \\
\hline 44 & Macko and Pereira (1990) & Antarctic margin (ODP 689) & unnamed & oceanic & $\mathrm{Pg}$ & mEocene1 & 48.70 & 40.39 & 44.55 & 16 \\
\hline 45 & Sadofsky and Bebout (2003) & California & Franciscan & oceanic & $\mathrm{Pg}$ & Eocene & 56.21 & 33.90 & 45.06 & 7 \\
\hline 46 & Meyers (unpubl.) & Arctic Ocean (ACES) & unnamed & oceanic-med & Pg & Eocene & 47.08 & 45.05 & 46.07 & 11 \\
\hline 47 & Sadofsky and Bebout (2004) & western Pacific (ODP 1149) & unnamed & oceanic & $\mathrm{Pg}$ & Paleoc-Eocene & 66.00 & 33.90 & 49.95 & 3 \\
\hline 48 & Hudson et al. (2008); Rowe (unpubl.) & Azerbaijan & Koun Fm & oceanic-med & $\mathrm{Pg}$ & IEocene & 56.00 & 48.70 & 52.35 & 41 \\
\hline 49 & Macko and Pereira (1990) & Antarctic margin (ODP 689) & unnamed & oceanic & $\mathrm{Pg}$ & lEocene & 54.18 & 52.76 & 53.47 & 6 \\
\hline 50 & $\begin{array}{l}\text { Hudson et al. (2008) and Rowe (un- } \\
\text { publ.) }\end{array}$ & Azerbaijan & Koun Fm & oceanic-med & $\mathrm{Pg}$ & Paleocene & 66.00 & 56.00 & 61.00 & 30 \\
\hline 51 & Meyers et al. (2009) & Demerara (ODP 1257-61) & unnamed & oceanic & Pg & Paleocene & 66.00 & 63.42 & 64.71 & 2 \\
\hline 52 & Martinez-Ruiz et al. (1994) & Spain & Agost section & shelf & $\mathrm{K} / \mathrm{T}$ & Maas-Paleocene & 66.50 & 65.50 & 66.00 & 12 \\
\hline 53 & Meyers et al. (2009) & Demerara (ODP 1257-61) & unnamed & oceanic & Cret & Camp-Maas & 83.60 & 66.00 & 74.80 & 9 \\
\hline 54 & Sadofsky and Bebout (2004) & western Pacific (ODP 1149) & unnamed & oceanic & Cret & Campanian & 83.60 & 72.10 & 77.85 & 3 \\
\hline 55 & Meyers et al. (2009) & Demerara (ODP 1257-61) & unnamed & oceanic-med & Cret & Santonian & 86.30 & 83.60 & 84.95 & 4 \\
\hline 56 & Junium and Arthur (2007) & Atlantic (ODP 1261) & unnamed & oceanic-med & Cret & Coniac-Santon & 90.00 & 85.95 & 87.98 & 23 \\
\hline 57 & Meyers et al. (2009) & Demerara (ODP 1257-61) & unnamed & oceanic-med & Cret & Coniacian & 89.80 & 86.30 & 88.05 & 5 \\
\hline 58 & Meyers et al. (2009) & ODP 1138 Kerguelen & unnamed & oceanic & Cret & uTuron-Santon & 91.95 & 84.19 & 88.07 & 27 \\
\hline 59 & Arnaboldi and Meyers (2006) & Newfoundland (ODP 1276) & unnamed & oceanic-med & Cret & Turonian & 93.41 & 89.80 & 91.61 & 5 \\
\hline 60 & Meyers et al. (2009) & Demerara (ODP 1257-61) & unnamed & oceanic-med & Cret & Turonian & 93.90 & 89.80 & 91.85 & 16 \\
\hline 61 & Jenkyns et al. (2007) & Morocco & unnamed & upwelling & Cret & Turonian & 93.90 & 91.46 & 92.68 & 46 \\
\hline 62 & Junium and Arthur (2007) & Atlantic (ODP 1261) & unnamed & oceanic-med & Cret & Cenom-Turon & 96.06 & 90.00 & 93.03 & 44 \\
\hline 63 & Arnaboldi and Meyers (2006) & Newfoundland (ODP 1276) & unnamed & oceanic-med & Cret & Cenom-Turon & 94.44 & 93.41 & 93.93 & 6 \\
\hline 64 & Jenkyns et al. (2007) & England & unnamed & epeiric & Cret & Cenom-Turon & 94.44 & 93.90 & 94.17 & 13 \\
\hline 65 & Jenkyns et al. (2007) & Italy-Furlo & Scaglia Bianca & oceanic-med & Cret & Cenom-Turon & 94.44 & 93.90 & 94.17 & 28 \\
\hline 66 & Jenkyns et al. (2007) & Italy-Gubbio & Scaglia Bianca & oceanic-med & Cret & Cenom-Turon & 94.44 & 93.90 & 94.17 & 49 \\
\hline 67 & Kuypers et al. (2004) & Atlantic (DSDP 367) & unnamed & oceanic-med & Cret & Cenomanian & 94.44 & 93.90 & 94.17 & 17 \\
\hline 68 & Ohkouchi et al. (2006) & Italy & Livello Bonarelli & oceanic-med & Cret & Cenom-Turon & 94.44 & 93.90 & 94.17 & 23 \\
\hline 69 & Junium and Arthur (2007) & Atlantic (ODP 1260) & unnamed & oceanic-med & Cret & Cenom-Turon & 96.06 & 92.92 & 94.49 & 56 \\
\hline 70 & Ohkouchi et al. (2006) & Italy & Scaglia Bianca & oceanic-med & Cret & Cenom & 94.98 & 94.44 & 94.71 & 21 \\
\hline 71 & Jenkyns et al. (2007) & Morocco & unnamed & upwelling & Cret & Cenom & 96.60 & 93.90 & 95.25 & 74 \\
\hline 72 & Meyers et al. (2009) & ODP 1138 Kerguelen & unnamed & oceanic & Cret & Cenom-ITuron & 99.85 & 91.95 & 95.90 & 16 \\
\hline 73 & Arnaboldi and Meyers (2006) & Newfoundland (ODP 1276) & unnamed & oceanic-med & Cret & Cenomanian & 99.85 & 94.44 & 97.15 & 4 \\
\hline 74 & Meyers et al. (2009) & Demerara (ODP 1257-61) & unnamed & oceanic-med & Cret & Cenomanian & 100.50 & 93.90 & 97.20 & 46 \\
\hline 75 & Rau et al. (1987) & South Atlantic (DSDP 530) & unnamed & oceanic-med & Cret & Aptian-Santon & 111.99 & 85.36 & 98.68 & 12 \\
\hline 76 & Arnaboldi and Meyers (2006) & Newfoundland (ODP 1276) & unnamed & oceanic-med & Cret & Albian-Cenom & 101.91 & 99.85 & 100.88 & 5 \\
\hline 77 & Rigby and Batts (1986) & Australia & Toolebuc & epeiric & Cret & Albian & 106.95 & 103.93 & 105.44 & 5 \\
\hline 78 & Meyers et al. (2009) & Demerara (ODP 1257-61) & unnamed & oceanic-med & Cret & Albian & 113.00 & 100.50 & 106.75 & 9 \\
\hline 79 & Sadofsky and Bebout (2003) & Baja California & unnamed & oceanic & Cret & uncertain & & & 107.46 & 5 \\
\hline 80 & Arnaboldi and Meyers (2006) & Newfoundland (ODP 1276) & unnamed & oceanic-med & Cret & uAptian-Albian & 114.02 & 101.91 & 107.97 & 21 \\
\hline
\end{tabular}


Table 1. Continued.

\begin{tabular}{|c|c|c|c|c|c|c|c|c|c|c|}
\hline Record & Source & Location & Formation & Setting & Period & Series & $\begin{array}{l}\text { Start } \\
(\mathrm{Ma})\end{array}$ & $\begin{array}{l}\text { Age } \\
\text { End } \\
\text { (Ma) }\end{array}$ & $\begin{array}{l}\text { Mid } \\
(\mathrm{Ma})\end{array}$ & $\begin{array}{l}\text { Sample } \\
n\end{array}$ \\
\hline 81 & Kuypers et al. (2002) & North Atlantic (ODP 1049C) & unnamed & oceanic-med & Cret & Albian & 111.99 & 111.49 & 111.74 & 6 \\
\hline 82 & Kuypers et al. (2004) & Atlantic (Cismon) & unnamed & oceanic-med & Cret & Aptian & 125.28 & 124.25 & 124.77 & 32 \\
\hline 83 & Rau et al. (1987) & North Atlantic (DSDP 603) & unnamed & oceanic-med & Cret & Valang-Barrem & 137.66 & 128.10 & 132.88 & 11 \\
\hline 84 & Rau et al. (1987) & North Atlantic (DSDP 367) & unnamed & oceanic-med & Cret & Valang-Hauter & 136.22 & 131.77 & 134.00 & 11 \\
\hline 85 & Saelen et al. (2000) & England & Kimmeridge Clay & epeiric & Jur & Kimmeridge & 155.50 & 152.31 & 153.91 & 13 \\
\hline 86 & Jenkyns et al. (2001) & Wales-MFB & Whitby Mudstone & epeiric & Jur & Toarcian & 182.70 & 176.89 & 179.80 & 71 \\
\hline 87 & Jenkyns et al. (2001) & England-WKB & Whitby Mudstone & epeiric & Jur & Toarcian & 182.70 & 179.21 & 180.96 & 33 \\
\hline 88 & Saelen et al. (2000) & England & Whitby Mudstone & epeiric & Jur & Toarcian & 182.70 & 180.38 & 181.54 & 15 \\
\hline 89 & Jenkyns et al. (2001) & England-HB & Whitby Mudstone & epeiric & Jur & Toarcian & 182.70 & 180.38 & 181.54 & 89 \\
\hline 90 & Jenkyns et al. (2001) & Italy & unnamed & epeiric & Jur & Toarcian & 182.70 & 180.38 & 181.54 & 74 \\
\hline 91 & Jenkyns et al. (2001) & England-WKB & Whitby Mudstone & epeiric & Jur & Pliensbachian & 185.15 & 182.70 & 183.93 & 10 \\
\hline 92 & Quan et al. (2008) & Germany & Lower Jurassic shales & epeiric & Jur & Hettang-Sinemur & & & 199.62 & 18 \\
\hline 94 & Paris et al. (2010) & England & Blue Lias & shelf & Jur & Hettangian & & & 200.91 & 44 \\
\hline 93 & Sephton et al. (2002) & Canada-Western & Fernie & epeiric & Jur & Hettangian & 202.12 & 200.27 & 201.20 & 3 \\
\hline 95 & Paris et al. (2010) & England & Lilstock & epeiric & Tri & Rhaetian & & & 204.17 & 20 \\
\hline 96 & Quan et al. (2008) & Germany & Keuper shales & epeiric & Tri & Rhaetian & & & 204.17 & 32 \\
\hline 97 & Sephton et al. (2002) & Canada-Western & Pardonet & shelf & Tri & Rhaetian & 210.09 & 202.12 & 206.11 & 6 \\
\hline 98 & Sephton et al. (2002) & Canada-Western & Pardonet & shelf & Tri & Norian & 227.67 & 210.09 & 218.88 & 8 \\
\hline 99 & Chicarelli et al. (1993) & Switzerland & $\begin{array}{l}\text { Scisti bituminosi (Serpi- } \\
\text { ano) }\end{array}$ & epeiric & Tri & Anisian-Ladinian & 242.20 & 241.00 & 241.60 & 4 \\
\hline 100 & Algeo and Rowe (unpubl.) & Switzerland & $\begin{array}{l}\text { Scisti bituminosi (Serpi- } \\
\text { ano) }\end{array}$ & epeiric & Tri & Anisian-Ladinian & 242.20 & 241.00 & 241.60 & 3 \\
\hline 101 & Algeo, Krystyn and Rowe (unpubl.) & India-Spiti & Mikin & shelf & Tri & Olenekian & 251.28 & 248.76 & 250.02 & 70 \\
\hline 102 & Algeo, Krystyn and Rowe (unpubl.) & India-Spiti & Mikin & shelf & Tri & Induan & 252.20 & 251.28 & 251.74 & 43 \\
\hline 103 & Algeo et al. (2007) & India-Kashmir & Khunamuh & shelf & Tri & Induan & 252.20 & 251.28 & 251.74 & 9 \\
\hline 104 & Algeo et al. (2012) & Canada-Arctic & Blind Fiord & shelf & Tri & Induan & 252.20 & 251.28 & 251.74 & 44 \\
\hline 105 & Algeo and Rowe (unpubl.) & China-East & Yinkeng & shelf & Tri & Induan & 252.20 & 251.74 & 251.97 & 30 \\
\hline 106 & Algeo and Rowe (unpubl.) & China-East & Dalong & shelf & Perm & Lopingian & 252.56 & 252.20 & 252.38 & 12 \\
\hline 107 & Algeo et al. (2012) & Canada-Arctic & Van Hauen & shelf & Perm & Lopingian & 252.56 & 252.20 & 252.38 & 11 \\
\hline 108 & Algeo et al. (2007) & India-Kashmir & Zewan & shelf & Perm & Lopingian & 252.91 & 252.20 & 252.56 & 8 \\
\hline 109 & Algeo, Krystyn and Rowe (unpubl.) & India-Spiti & Kuling & shelf & Perm & Lopingian & 252.91 & 252.20 & 252.56 & 22 \\
\hline 110 & Algeo and Rowe (unpubl.) & Kansas & Eudora & epeiric & Penn & Missourian & 303.31 & 302.82 & 303.07 & 83 \\
\hline 111 & Algeo and Rowe (unpubl.) & Kansas & Wea & epeiric & Penn & Missourian & 303.83 & 303.31 & 303.57 & 34 \\
\hline 112 & Algeo et al. (2008) & Kansas & Muncie Creek & epeiric & Penn & Missourian & 304.46 & 303.83 & 304.15 & 42 \\
\hline 113 & Algeo et al. (2008) & Kansas & Stark & epeiric & Penn & Missourian & 305.73 & 305.10 & 305.42 & 37 \\
\hline 114 & Algeo et al. (2008) & Kansas & Hushpuckney & epeiric & Penn & Missourian & 306.37 & 305.73 & 306.05 & 45 \\
\hline 115 & Rowe (unpubl.) & Texas & Smithwick & shelf & Penn & Atokan & 310.94 & 307.00 & 308.97 & 149 \\
\hline 116 & Johnson et al. (2009) & Alaska & Lisburne (shallow facies) & shelf & Miss & unknown & 338.09 & 319.33 & 328.71 & 9 \\
\hline 117 & Rowe (unpubl.) & Texas (Blakely-Wise Co) & Barnett & shelf & Miss & Visean & 337.25 & 323.08 & 330.17 & 128 \\
\hline 118 & Rowe (unpubl.) & Texas (RTC-Pecos Co) & Barnett & shelf & Miss & Visean & 337.25 & 323.08 & 330.17 & 177 \\
\hline 119 & Rowe (unpubl.) & Texas (Johanson-McCulloch Co) & Barnett & shelf & Miss & Visean & 337.25 & 323.08 & 330.17 & 24 \\
\hline 120 & Rowe (unpubl.) & Texas (Lee-Brown Co) & Barnett & shelf & Miss & Visean & 337.25 & 323.08 & 330.17 & 36 \\
\hline 121 & Rowe (unpubl.) & Texas (Locker-San Saba Co) & Barnett & shelf & Miss & Visean & 337.25 & 323.08 & 330.17 & 99 \\
\hline 122 & Johnson et al. (2009) & Alaska & Lisburne-Kuna Fm & shelf & Miss & Tourn-Visean & 350.83 & 330.53 & 340.68 & 17 \\
\hline 123 & Algeo and Sauer (unpubl.) & Ohio-Kentucky & Sunbury & epeiric & Miss & Tournaisian & 358.72 & 356.97 & 357.85 & 40 \\
\hline 124 & Caplan and Bustin (1998) & Alberta & Exshaw & epeiric & Miss & Tournaisian & 359.60 & 357.85 & 358.73 & 21 \\
\hline 125 & Algeo and Sauer (unpubl.) & Ohio-Kentucky & Ohio Shale & epeiric & Dev & Famennian & 362.20 & 360.46 & 361.33 & 20 \\
\hline 126 & Meyers (unpubl.) & Alberta & Exshaw & epeiric & Dev & Famennian & 362.20 & 360.46 & 361.33 & 28 \\
\hline 127 & Caplan and Bustin (1998) & Alberta & Exshaw & epeiric & Dev & Famennian & 362.20 & 360.46 & 361.33 & 16 \\
\hline 128 & Calvert et al. (1996) & Indiana & New Albany & epeiric & Dev & Famennian & 369.16 & 368.29 & 368.73 & 71 \\
\hline 129 & de la Rue et al. (2007) & Indiana & New Albany & epeiric & Dev & Famennian & 372.20 & 371.77 & 371.99 & 10 \\
\hline 130 & Levman and von Bitter (2002) & Ontario & Long Rapids & epeiric & Dev & Frasn-Famen & 373.66 & 370.90 & 372.28 & 24 \\
\hline 131 & de la Rue et al. (2007) & Indiana & New Albany & epeiric & Dev & Frasnian & 372.69 & 372.20 & 372.45 & 20 \\
\hline 132 & Sageman (unpubl.) & New York & Geneseo-Rhinestreet & epeiric & Dev & Frasnian & 382.41 & 375.60 & 379.01 & 16 \\
\hline 133 & Sageman (unpubl.) & New York & upper Hamilton & epeiric & Dev & Givetian & 387.90 & 382.41 & 385.16 & 46 \\
\hline 134 & Sageman (unpubl.) & New York & Marcellus & epeiric & Dev & Eifelian & 390.84 & 387.90 & 389.37 & 58 \\
\hline 135 & Bauersachs et al. (2009) & Poland & Bardo/Lwr Graptolitic & epeiric & Sil & Llandov & 440.80 & 433.11 & 436.96 & 8 \\
\hline 136 & LaPorte et al. (2009) & Nevada-Monitor Range & unnamed & epeiric & Ord & Katian-Hirnant & 446.65 & 443.80 & 445.23 & 68 \\
\hline 137 & LaPorte et al. (2009) & Nevada-Vinini Creek & unnamed & epeiric & Ord & Katian-Hirnant & 447.80 & 443.80 & 445.80 & 116 \\
\hline 138 & Algeo, Lev and Rowe (unpubl.) & Wales & Llandeilo-Caradoc Shales & epeiric & Ord & Sandbian & 458.52 & 455.33 & 456.93 & 41 \\
\hline 139 & Algeo, Lev and Rowe (unpubl.) & Wales & Caerhys Shale & epeiric & Ord & Daping-Darriwill. & 462.23 & 459.76 & 461.00 & 23 \\
\hline 140 & Algeo, Lev and Rowe (unpubl.) & Wales & Aber Mawr Shale & epeiric & Ord & Dapingian & 465.94 & 463.47 & 464.71 & 28 \\
\hline 141 & Algeo and Rowe (unpubl.) & Germany & Lwr Didymograptus Shale & epeiric & Ord & Floian & 473.62 & 470.23 & 471.93 & 1 \\
\hline 142 & Algeo and Rowe (unpubl.) & Utah & Wheeler & epeiric & $\mathrm{Cam}$ & Delam-Marjum & 503.00 & 502.00 & 502.50 & 18 \\
\hline 143 & Jiang (unpubl.) & China & Shuijingtuo & epeiric & $\mathrm{Cam}$ & Tommotian & 522.24 & 517.83 & 520.04 & 9 \\
\hline 144 & Jiang (unpubl.) & China & Yanjiahe & epeiric & Cam & $\begin{array}{l}\text { Nemakit- } \\
\text { Daldynian }\end{array}$ & 541.00 & 522.24 & 531.62 & 76 \\
\hline 145 & Jiang (unpubl.) & China & Dengying (BMT Member) & epeiric & Neopr & & 545.00 & 541.00 & 543.00 & 2 \\
\hline 146 & Jiang (unpubl.) & China & Dengying (SBT Member) & epeiric & Neopr & & 548.00 & 545.00 & 546.50 & 24 \\
\hline 147 & Jiang (unpubl.) & China & Dengying (HMJ Member) & epeiric & Neopr & & 551.00 & 548.00 & 549.50 & 7 \\
\hline 148 & Algeo and Rowe (unpubl.) & China & Doushantuo (Member 4) & epeiric & Neopr & & 560.00 & 551.00 & 555.50 & 9 \\
\hline 149 & Jiang (unpubl.) & China & Doushantuo (Member 4) & epeiric & Neopr & & 560.00 & 551.00 & 555.50 & 39 \\
\hline 150 & Jiang (unpubl.) & China & Doushantuo (Member 3) & epeiric & Neopr & & 600.00 & 560.00 & 580.00 & 11 \\
\hline 151 & Jiang (unpubl.) & China & Doushantuo (Member 2) & epeiric & Neopr & & 632.00 & 600.00 & 616.00 & 86 \\
\hline 152 & Jiang (unpubl.) & China & Doushantuo (Member 1) & epeiric & Neopr & & 635.00 & 632.00 & 633.50 & 7 \\
\hline 153 & Jiang (unpubl.) & China & Xiangmeng & epeiric & Neopr & & 663.00 & 654.00 & 658.50 & 34 \\
\hline
\end{tabular}


Table 1. Continued.

\begin{tabular}{|c|c|c|c|c|c|c|c|c|c|c|c|c|c|}
\hline \multirow{3}{*}{ Record } & Ele & & & & & C-Isotopes & & & & & N-Isotopes & & \\
\hline & TOC & $\mathrm{N}$ & $\mathrm{C}: \mathrm{N}$ & Min & & Percentiles & & Max & Min & & Percentiles & & $\operatorname{Max}$ \\
\hline & $(\%)$ & $(\%)$ & $(\mathrm{mol})$ & & 16th & 50 th & 84th & & & 16th & 50 th & 84th & \\
\hline 1 & 6.06 & 0.48 & 14.90 & -22.10 & -21.70 & -21.40 & -21.10 & -20.20 & 4.30 & 4.90 & 5.70 & 6.47 & 6.80 \\
\hline 2 & 5.01 & 0.36 & 16.20 & -22.94 & -21.70 & -21.12 & -20.35 & -19.40 & -0.24 & 0.79 & 1.77 & 2.98 & 3.80 \\
\hline 3 & 0.12 & 0.22 & 0.60 & -24.60 & -23.40 & -22.90 & -22.24 & -21.00 & 1.00 & 2.94 & 4.25 & 5.90 & 7.30 \\
\hline 4 & 7.90 & & & -23.00 & -21.34 & -20.50 & -19.90 & -18.60 & 3.40 & 5.40 & 6.60 & 7.90 & 14.00 \\
\hline 5 & 0.51 & 0.06 & 9.70 & -26.03 & -25.26 & -24.30 & -23.02 & -22.22 & 0.07 & 2.29 & 4.47 & 5.19 & 5.81 \\
\hline 6 & 6.00 & 0.28 & 24.60 & -25.92 & -25.00 & -23.83 & -23.30 & -21.39 & -2.50 & -1.84 & 0.18 & 4.64 & 6.23 \\
\hline 7 & & & & -23.23 & -22.48 & -22.05 & -21.49 & -20.66 & -5.12 & -2.30 & 0.07 & 4.04 & 5.15 \\
\hline 8 & 1.48 & 0.16 & 10.80 & -26.30 & -24.98 & -24.00 & -23.20 & -22.50 & 3.50 & 3.82 & 4.55 & 5.97 & 6.60 \\
\hline 9 & 11.16 & & & & & & & & -2.72 & -2.46 & -1.67 & 3.97 & 4.93 \\
\hline 10 & 3.48 & 0.27 & 15.30 & & & & & & 4.00 & 6.19 & 6.80 & 7.38 & 9.57 \\
\hline 11 & 3.70 & & & -23.90 & -23.40 & -22.30 & -21.15 & -19.30 & -1.10 & 0.28 & 1.30 & 2.05 & 4.10 \\
\hline 12 & 1.58 & 0.18 & 10.40 & -26.60 & -25.82 & -24.80 & -24.26 & -21.50 & 3.60 & 4.09 & 4.30 & 5.61 & 6.00 \\
\hline 13 & 0.10 & 0.03 & 4.00 & -24.50 & -23.10 & -22.50 & -22.00 & -21.20 & 2.20 & 3.10 & 3.80 & 4.40 & 4.90 \\
\hline 14 & 0.11 & 0.12 & 1.00 & -27.00 & -25.00 & -24.00 & -22.32 & -20.60 & 2.00 & 3.82 & 5.00 & 6.18 & 7.40 \\
\hline 15 & 0.13 & 0.12 & 1.20 & -26.00 & -23.74 & -23.10 & -22.20 & -21.60 & 1.00 & 2.90 & 3.80 & 4.50 & 6.10 \\
\hline 16 & 0.19 & 0.03 & 6.40 & -24.50 & -23.51 & -23.10 & -22.22 & -21.70 & 4.70 & 4.92 & 5.50 & 5.74 & 6.30 \\
\hline 17 & 0.06 & 0.04 & 2.00 & -27.20 & -26.41 & -25.60 & -23.04 & -19.70 & 1.10 & 1.70 & 2.40 & 3.70 & 5.60 \\
\hline 18 & 0.19 & 0.05 & 4.40 & -26.70 & -25.73 & -24.30 & -23.07 & -22.10 & 2.20 & 2.90 & 3.60 & 4.60 & 5.00 \\
\hline 19 & 0.18 & 0.04 & 4.90 & -26.10 & -23.50 & -22.70 & -22.30 & -21.70 & 2.40 & 3.16 & 4.30 & 4.70 & 6.70 \\
\hline 20 & 0.07 & 0.03 & 2.40 & -28.50 & -26.95 & -24.70 & -23.24 & -21.10 & 1.50 & 2.48 & 3.15 & 3.99 & 4.70 \\
\hline 21 & 0.07 & 0.18 & 0.40 & -29.40 & -28.88 & -27.30 & -26.10 & -23.60 & 3.00 & 3.62 & 5.00 & 6.54 & 8.70 \\
\hline 22 & 5.85 & 0.64 & 10.70 & -22.93 & -22.23 & -21.55 & -21.30 & -19.83 & 1.50 & 4.96 & 6.60 & 7.50 & 14.00 \\
\hline 23 & 0.83 & 0.10 & 9.70 & -27.01 & -25.65 & -24.05 & -23.19 & -20.65 & -0.34 & 2.00 & 3.10 & 3.78 & 4.16 \\
\hline 24 & 0.07 & 0.03 & 3.40 & -28.10 & -27.70 & -25.90 & -24.69 & -23.40 & 2.80 & 3.08 & 3.70 & 4.20 & 4.90 \\
\hline 25 & 0.37 & 0.05 & 9.30 & -26.60 & -23.60 & -23.20 & -22.70 & -21.10 & 2.30 & 3.30 & 4.20 & 4.90 & 7.20 \\
\hline 26 & 0.06 & 0.22 & 0.30 & -29.80 & -29.62 & -27.90 & -26.77 & -24.40 & 1.70 & 3.16 & 3.90 & 5.43 & 6.00 \\
\hline 27 & 0.16 & 0.05 & 3.40 & -23.70 & -23.42 & -23.00 & -22.60 & -22.00 & 3.80 & 4.37 & 5.30 & 6.00 & 6.10 \\
\hline 28 & 0.07 & 0.17 & 0.50 & -29.10 & -28.81 & -28.35 & -27.94 & -27.70 & 5.10 & 5.10 & 5.15 & 5.46 & 5.70 \\
\hline 29 & 0.04 & 0.03 & 1.60 & -29.10 & -28.70 & -27.30 & -26.86 & -25.90 & 2.80 & 2.83 & 3.20 & 3.42 & 4.10 \\
\hline 30 & 1.39 & 0.13 & 12.50 & -28.22 & -26.83 & -26.32 & -24.46 & -23.44 & -1.30 & 1.22 & 1.89 & 3.25 & 4.01 \\
\hline 31 & 0.10 & 0.03 & 3.90 & -23.70 & -23.56 & -22.80 & -22.54 & -22.00 & 4.50 & 4.93 & 5.35 & 5.70 & 6.00 \\
\hline 32 & 1.96 & 0.14 & 16.30 & -28.56 & -27.30 & -26.57 & -25.22 & -23.68 & -1.37 & 0.02 & 1.05 & 2.52 & 4.20 \\
\hline 33 & 0.12 & 0.04 & 3.70 & -23.40 & -23.28 & -22.70 & -22.40 & -22.10 & 5.20 & 5.30 & 5.60 & 5.70 & 6.00 \\
\hline 34 & 0.08 & 0.04 & 2.40 & -29.60 & -28.90 & -28.45 & -28.05 & -26.30 & 2.50 & 2.75 & 3.60 & 4.67 & 5.20 \\
\hline 35 & 0.08 & 0.10 & 1.00 & -28.80 & -28.64 & -28.50 & -28.16 & -26.60 & 5.20 & 5.20 & 5.30 & 5.48 & 5.60 \\
\hline 36 & 0.21 & 0.05 & 4.60 & -24.80 & -24.26 & -23.85 & -23.20 & -21.20 & 3.00 & 3.30 & 3.55 & 4.40 & 5.00 \\
\hline 37 & 1.01 & 0.11 & 10.70 & -28.00 & -26.87 & -25.56 & -24.28 & -23.13 & -0.97 & 0.08 & 1.56 & 2.87 & 4.52 \\
\hline 38 & 0.11 & 0.06 & 2.10 & -29.30 & -29.14 & -28.60 & -28.24 & -27.90 & 2.70 & 3.70 & 4.40 & 4.58 & 5.20 \\
\hline 39 & 3.07 & & & -29.55 & -28.68 & -26.20 & -25.64 & -23.25 & -0.30 & 0.79 & 1.60 & 2.25 & 3.60 \\
\hline 40 & 0.33 & 0.06 & 6.40 & -29.92 & -27.49 & -26.04 & -25.15 & -21.40 & -0.25 & 3.18 & 5.07 & 8.02 & 25.47 \\
\hline 41 & 0.12 & 0.07 & 2.10 & -29.40 & -27.94 & -27.20 & -25.10 & -25.00 & 3.30 & 4.30 & 5.15 & 5.56 & 6.00 \\
\hline 42 & 0.09 & 0.09 & 1.20 & -27.90 & -26.98 & -26.55 & -25.74 & -25.60 & 2.90 & 2.92 & 3.95 & 5.76 & 5.90 \\
\hline 43 & 0.25 & 0.06 & 4.90 & -28.36 & -26.25 & -25.12 & -24.62 & -18.14 & 0.08 & 4.01 & 5.77 & 7.86 & 12.29 \\
\hline 44 & 0.08 & 0.05 & 1.90 & -26.60 & -26.16 & -25.85 & -25.44 & -25.10 & 3.20 & 3.64 & 4.50 & 5.94 & 7.50 \\
\hline 45 & 0.43 & 0.05 & 10.80 & -25.80 & -25.40 & -25.25 & -24.98 & -24.50 & 1.40 & 2.36 & 2.80 & 3.02 & 3.40 \\
\hline 46 & 3.10 & 0.12 & 24.90 & -28.60 & -28.48 & -27.70 & -27.28 & -27.00 & -2.40 & -2.18 & -1.80 & -1.56 & -1.50 \\
\hline 47 & 0.08 & 0.01 & 33.94 & -24.40 & -24.02 & -23.20 & -22.93 & -22.80 & 3.70 & 3.76 & 3.90 & 4.38 & 4.60 \\
\hline 48 & 0.38 & 0.07 & 6.30 & -28.15 & -27.03 & -25.45 & -24.83 & -22.50 & 2.01 & 3.90 & 5.43 & 7.35 & 14.81 \\
\hline 49 & 0.13 & 0.04 & 3.70 & -27.30 & -26.82 & -26.50 & -26.34 & -26.10 & 5.30 & 5.86 & 6.40 & 7.20 & 7.60 \\
\hline 50 & 0.19 & 0.04 & 5.50 & -27.39 & -26.55 & -25.86 & -25.31 & -24.32 & -1.86 & 3.18 & 4.43 & 5.33 & 6.99 \\
\hline 51 & 0.45 & 0.03 & 16.30 & -29.00 & -28.90 & -28.70 & -28.50 & -28.40 & 4.20 & 4.22 & 4.25 & 4.28 & 4.30 \\
\hline 52 & & & & -25.70 & -24.73 & -24.20 & -23.85 & -23.15 & 3.90 & 4.17 & 4.46 & 5.16 & 5.60 \\
\hline 53 & 0.20 & 0.02 & 12.90 & -29.00 & -28.79 & -27.70 & -27.46 & -26.70 & 2.30 & 3.41 & 3.90 & 4.14 & 4.40 \\
\hline 54 & 0.08 & 0.01 & 28.07 & -25.60 & -25.57 & -25.50 & -25.16 & -25.00 & 4.50 & 4.56 & 4.70 & 4.84 & 4.90 \\
\hline 55 & 10.04 & 0.35 & 33.00 & -29.20 & -28.77 & -27.85 & -27.04 & -26.70 & -2.30 & -2.16 & -2.00 & -1.79 & -1.60 \\
\hline 56 & 20.05 & 0.68 & 34.40 & -28.90 & -28.00 & -27.10 & -26.11 & -22.75 & -2.80 & -2.07 & -1.30 & 0.30 & 1.20 \\
\hline 57 & 11.42 & 0.39 & 34.30 & -27.80 & -27.74 & -27.60 & -27.15 & -26.70 & 1.40 & 1.66 & 3.40 & 3.70 & 3.70 \\
\hline 58 & 1.81 & 0.06 & 34.90 & -29.70 & -27.05 & -26.30 & -25.60 & -24.70 & -7.80 & -3.80 & -1.00 & 0.77 & 3.00 \\
\hline 59 & 1.51 & 0.10 & 18.30 & -26.52 & -26.06 & -25.65 & -25.28 & -25.14 & -2.51 & -1.71 & -0.15 & 1.02 & 2.30 \\
\hline 60 & 10.62 & 0.41 & 30.10 & -28.30 & -27.78 & -27.50 & -27.10 & -26.20 & -3.50 & -3.02 & -2.05 & -1.24 & 2.80 \\
\hline 61 & 7.20 & & & -27.60 & -27.08 & -26.65 & -25.84 & -24.80 & -2.26 & -1.75 & -1.45 & -0.90 & -0.75 \\
\hline 62 & 20.28 & 0.60 & 39.40 & -28.80 & -28.04 & -27.10 & -25.10 & -22.10 & -2.90 & -2.60 & -1.65 & -0.59 & 1.33 \\
\hline 63 & 3.74 & 0.15 & 28.20 & -26.54 & -26.16 & -25.12 & -23.93 & -23.82 & -2.70 & -2.60 & -2.17 & -1.83 & -0.65 \\
\hline 64 & 2.30 & & & -24.85 & -24.73 & -24.13 & -23.19 & -22.90 & -3.72 & -3.35 & -2.89 & -1.98 & -1.92 \\
\hline 65 & 7.40 & & & -27.20 & -26.46 & -26.10 & -24.88 & -23.20 & -4.87 & -3.70 & -3.38 & -2.88 & -1.72 \\
\hline 66 & 8.60 & & & -25.30 & -24.35 & -23.40 & -22.95 & -22.60 & -5.74 & -3.95 & -3.14 & -2.52 & -1.60 \\
\hline 67 & 19.00 & & & -28.30 & -27.47 & -25.95 & -21.90 & -21.40 & -2.27 & -1.91 & -1.65 & -0.86 & 0.20 \\
\hline 68 & 11.10 & 0.40 & 32.38 & & & & & & -2.68 & -2.42 & -1.78 & -1.45 & 3.00 \\
\hline 69 & 22.57 & 0.75 & 35.10 & -28.90 & -28.50 & -27.80 & -26.79 & -23.20 & -2.75 & -1.85 & -1.15 & -0.63 & 0.03 \\
\hline 70 & 10.89 & 0.42 & 30.60 & & & & & & -1.22 & -0.22 & 1.22 & 2.04 & 2.55 \\
\hline 71 & 7.50 & & & -28.10 & -27.20 & -25.10 & -24.40 & -23.50 & -2.73 & -2.27 & -1.69 & -1.08 & -0.70 \\
\hline 72 & 5.16 & 0.20 & 30.40 & -27.10 & -26.64 & -25.60 & -24.71 & -23.60 & -4.10 & -3.90 & -2.55 & 0.38 & 3.30 \\
\hline 73 & 0.79 & 0.05 & 18.60 & -27.25 & -27.01 & -26.36 & -22.63 & -19.57 & -3.17 & -2.12 & 0.05 & 1.15 & 1.20 \\
\hline 74 & 10.17 & 0.34 & 34.50 & -29.70 & -29.18 & -28.60 & -27.46 & -23.90 & -4.20 & -2.60 & -1.90 & -1.22 & 0.30 \\
\hline 75 & 5.80 & 0.26 & 26.00 & -27.60 & -27.60 & -27.05 & -26.85 & -26.30 & -2.68 & -2.10 & 0.13 & 3.62 & 5.72 \\
\hline 76 & 0.82 & 0.06 & 17.20 & -28.28 & -27.54 & -25.56 & -25.13 & -25.13 & -2.45 & -2.15 & 1.40 & 1.83 & 2.60 \\
\hline 77 & & 0.40 & & & & & & & -2.50 & -2.37 & -0.70 & 0.29 & 1.70 \\
\hline 78 & 3.84 & 0.15 & 30.00 & -29.00 & -28.64 & -28.50 & -27.29 & -23.40 & -2.00 & -1.30 & -0.90 & 0.09 & 3.40 \\
\hline 79 & 0.16 & 0.03 & 5.80 & -28.80 & -25.41 & -24.65 & -22.74 & -21.90 & 0.10 & 1.25 & 1.90 & 2.61 & 2.80 \\
\hline 80 & 1.65 & 0.08 & 22.60 & -27.28 & -26.72 & -24.29 & -23.21 & -21.85 & -2.09 & -1.83 & -0.95 & 1.38 & 2.36 \\
\hline
\end{tabular}


Table 1. Continued.

\begin{tabular}{|c|c|c|c|c|c|c|c|c|c|c|c|c|c|}
\hline \multirow[b]{2}{*}{ Record } & \multicolumn{3}{|c|}{ Elemental } & \multirow[b]{2}{*}{ Min } & \multirow[b]{2}{*}{ 16th } & \multicolumn{2}{|l|}{ C-Isotopes } & & & & N-Isotopes & & \\
\hline & $\begin{array}{l}\text { TOC } \\
(\%)\end{array}$ & $\begin{array}{l}\mathrm{N} \\
(\%)\end{array}$ & $\begin{array}{l}\mathrm{C}: \mathrm{N} \\
(\mathrm{mol})\end{array}$ & & & $\begin{array}{l}\text { Percentiles } \\
\text { 50th }\end{array}$ & 84th & Max & Min & 16th & $\begin{array}{l}\text { Percentiles } \\
\text { 50th }\end{array}$ & 84th & $\operatorname{Max}$ \\
\hline 81 & 3.68 & 0.09 & 47.40 & -24.30 & -24.16 & -21.70 & -20.03 & -17.20 & -5.45 & -4.93 & -3.12 & -1.33 & -1.31 \\
\hline 82 & 0.60 & & & -28.70 & -26.27 & -25.20 & -24.34 & -22.70 & -2.58 & -1.89 & 0.05 & 1.29 & 2.10 \\
\hline 83 & 1.48 & 0.07 & 26.30 & -26.70 & -26.20 & -25.30 & -24.76 & -24.40 & -0.66 & 0.83 & 2.07 & 2.81 & 3.75 \\
\hline 84 & 1.12 & 0.06 & 20.50 & -28.30 & -28.04 & -27.70 & -27.30 & -27.00 & -1.67 & -0.36 & 2.33 & 3.05 & 5.02 \\
\hline 85 & 14.67 & 0.66 & 25.90 & -26.80 & -26.04 & -24.00 & -21.60 & -21.30 & 0.49 & 0.95 & 1.53 & 2.11 & 2.58 \\
\hline 86 & 1.14 & & & -30.86 & -28.66 & -27.24 & -25.61 & -24.10 & -3.09 & -1.81 & -0.96 & 0.39 & 2.38 \\
\hline 87 & 0.91 & & & -30.85 & -28.05 & -25.85 & -25.12 & -24.53 & -2.89 & -1.72 & -1.04 & 0.11 & 1.50 \\
\hline 88 & 6.91 & 0.26 & 30.80 & -31.30 & -31.03 & -28.60 & -26.95 & -26.60 & 1.55 & 1.66 & 2.29 & 2.65 & 2.84 \\
\hline 89 & 5.59 & & & -32.20 & -30.96 & -28.79 & -26.76 & -25.62 & -3.57 & -2.09 & -1.09 & 0.33 & 1.99 \\
\hline 90 & 1.28 & & & -34.00 & -32.80 & -31.90 & -31.20 & -28.60 & -2.88 & -1.50 & -0.63 & 1.33 & 3.80 \\
\hline 91 & 0.51 & & & -27.75 & -27.12 & -26.90 & -25.92 & -25.43 & -2.12 & -1.37 & -0.76 & -0.17 & 0.04 \\
\hline 92 & 1.57 & & & -29.90 & -29.36 & -28.45 & -27.70 & -27.60 & 1.10 & 1.24 & 1.60 & 1.93 & 2.20 \\
\hline 94 & 2.80 & & & -29.90 & -29.60 & -29.05 & -28.49 & -26.80 & 1.70 & 2.10 & 2.60 & 3.01 & 3.30 \\
\hline 93 & 2.19 & 0.49 & 5.20 & -32.00 & -31.94 & -31.80 & -31.39 & -31.20 & 1.96 & 2.00 & 2.09 & 2.95 & 3.35 \\
\hline 95 & 1.50 & & & -29.70 & -28.75 & -26.70 & -26.00 & -25.70 & 3.30 & 3.50 & 3.60 & 4.19 & 5.10 \\
\hline 96 & 0.41 & & & -29.60 & -27.50 & -26.10 & -25.40 & -25.10 & 0.10 & 0.40 & 0.85 & 1.21 & 2.10 \\
\hline 97 & 1.66 & 0.26 & 7.50 & -31.90 & -30.70 & -30.05 & -28.94 & -28.70 & -0.62 & 0.03 & 0.82 & 1.61 & 1.89 \\
\hline 98 & 1.17 & 0.17 & 8.20 & -30.90 & -30.89 & -30.70 & -30.04 & -29.70 & 2.25 & 2.54 & 3.12 & 4.59 & 4.98 \\
\hline 99 & & & & & -31.59 & -31.56 & -31.53 & & & -4.11 & -3.97 & -3.83 & \\
\hline 100 & 18.53 & 0.38 & 34.50 & -30.10 & -30.09 & -30.02 & -29.95 & -29.94 & -4.93 & -4.61 & -3.94 & -3.79 & -3.72 \\
\hline 101 & 0.09 & 0.01 & 7.00 & -31.56 & -30.55 & -27.97 & -26.86 & -25.08 & 0.66 & 2.24 & 3.02 & 3.55 & 4.32 \\
\hline 102 & 0.63 & 0.06 & 11.80 & -29.58 & -28.87 & -28.20 & -27.44 & -26.70 & 2.18 & 2.68 & 3.03 & 3.60 & 4.46 \\
\hline 103 & 0.27 & 0.07 & 4.30 & -27.86 & -27.31 & -25.98 & -25.31 & -24.69 & 1.34 & 1.77 & 2.13 & 2.34 & 2.42 \\
\hline 104 & 0.16 & 0.09 & 2.10 & -31.46 & -30.75 & -29.03 & -27.60 & -25.38 & 4.49 & 4.83 & 5.34 & 6.02 & 6.38 \\
\hline 105 & 0.08 & 0.11 & 0.80 & -26.09 & -25.54 & -24.80 & -24.21 & -22.24 & -0.49 & 0.17 & 0.32 & 0.42 & 1.79 \\
\hline 106 & 1.23 & 0.19 & 7.60 & -28.09 & -27.41 & -26.57 & -25.42 & -24.12 & -1.19 & -0.01 & 0.24 & 0.65 & 1.10 \\
\hline 107 & 0.17 & 0.01 & 14.30 & -27.36 & -26.85 & -26.46 & -26.15 & -25.75 & 4.00 & 4.21 & 4.88 & 5.55 & 5.88 \\
\hline 108 & 0.20 & 0.02 & 9.30 & -26.95 & -26.69 & -24.21 & -23.67 & -23.51 & 1.55 & 1.71 & 2.19 & 3.44 & 4.22 \\
\hline 109 & 0.95 & 0.09 & 12.70 & -27.63 & -24.44 & -24.04 & -23.80 & -23.57 & 3.05 & 3.25 & 3.60 & 3.96 & 4.67 \\
\hline 110 & 5.77 & 0.39 & 17.10 & -27.65 & -26.35 & -25.44 & -24.76 & -23.47 & -0.44 & 3.36 & 5.33 & 7.23 & 10.52 \\
\hline 111 & 0.79 & 0.11 & 8.30 & -24.28 & -23.99 & -23.84 & -23.54 & -23.35 & 1.97 & 2.44 & 3.46 & 4.24 & 5.06 \\
\hline 112 & 11.54 & 0.66 & 20.40 & -27.98 & -27.70 & -26.80 & -25.58 & -24.45 & 4.26 & 5.15 & 5.88 & 11.77 & 12.90 \\
\hline 113 & 10.87 & 0.59 & 21.50 & -27.87 & -27.20 & -26.72 & -25.73 & -25.18 & 4.49 & 5.37 & 5.69 & 6.03 & 7.05 \\
\hline 114 & 14.34 & 0.68 & 24.50 & -29.11 & -28.67 & -27.91 & -26.89 & -25.34 & 4.05 & 4.64 & 5.23 & 9.17 & 13.39 \\
\hline 115 & 1.52 & 0.15 & 11.80 & -25.72 & -24.77 & -24.25 & -23.83 & -22.71 & 2.27 & 4.18 & 4.76 & 5.20 & 5.52 \\
\hline 116 & 2.88 & 0.24 & 14.00 & -31.50 & -31.29 & -30.70 & -29.25 & -27.00 & 8.90 & 9.50 & 10.40 & 11.30 & 12.00 \\
\hline 117 & 2.92 & 0.34 & 10.00 & -29.54 & -29.08 & -28.26 & -27.64 & -27.05 & 5.98 & 9.65 & 10.42 & 10.90 & 11.77 \\
\hline 118 & 2.89 & 0.29 & 11.60 & -30.91 & -29.27 & -28.28 & -27.50 & -24.27 & 3.91 & 5.48 & 6.10 & 7.27 & 8.74 \\
\hline 119 & 2.36 & 0.14 & 19.60 & -29.62 & -29.37 & -28.80 & -28.22 & -27.76 & 3.92 & 5.35 & 6.52 & 7.69 & 10.70 \\
\hline 120 & 5.20 & 0.26 & 23.30 & -30.33 & -30.13 & -29.55 & -28.60 & -24.77 & 3.53 & 7.12 & 9.05 & 10.35 & 11.48 \\
\hline 121 & 4.52 & 0.24 & 21.50 & -30.91 & -30.19 & -29.61 & -29.01 & -27.32 & 3.24 & 6.91 & 8.89 & 10.76 & 11.80 \\
\hline 122 & 1.34 & 0.20 & 7.82 & -30.24 & -30.08 & -29.66 & -29.45 & -29.33 & 6.40 & 7.40 & 8.60 & 9.80 & 11.20 \\
\hline 123 & 9.90 & 0.42 & 27.80 & -30.46 & -30.38 & -30.18 & -29.91 & -29.72 & -0.59 & -0.06 & 1.37 & 2.14 & 2.47 \\
\hline 124 & 7.94 & 0.27 & 34.05 & -28.75 & -28.57 & -28.37 & -28.14 & -27.80 & -0.37 & -0.15 & 0.56 & 1.74 & 2.97 \\
\hline 125 & 8.13 & 0.28 & 33.70 & -29.90 & -29.61 & -28.37 & -27.58 & -27.49 & 0.16 & 0.64 & 1.84 & 4.03 & 4.55 \\
\hline 126 & 5.68 & & & -28.75 & -28.52 & -28.25 & -27.42 & -26.76 & -0.37 & -0.07 & 1.15 & 1.90 & 2.97 \\
\hline 127 & 9.94 & 0.00 & & -28.50 & -28.01 & -27.39 & -26.82 & -26.07 & 0.10 & 1.23 & 2.09 & 2.98 & 3.70 \\
\hline 128 & 5.38 & 0.26 & 24.60 & -30.00 & -29.60 & -28.85 & -27.30 & -23.50 & -1.20 & -0.10 & 0.70 & 2.20 & 2.90 \\
\hline 129 & 9.57 & 0.30 & 37.00 & -29.00 & -28.74 & -28.42 & -28.09 & -27.86 & -0.05 & 0.11 & 0.22 & 0.55 & 0.76 \\
\hline 130 & 6.26 & & & -28.61 & -27.63 & -27.28 & -26.63 & -25.96 & -2.26 & -2.01 & -1.67 & 1.02 & 1.73 \\
\hline 131 & 0.97 & 0.11 & 10.70 & -30.14 & -29.90 & -29.44 & -29.16 & -29.07 & 1.01 & 1.27 & 1.72 & 1.95 & 2.00 \\
\hline 132 & 2.66 & 0.16 & 19.50 & -31.00 & -29.95 & -29.35 & -28.45 & -27.05 & -3.75 & -1.70 & -0.50 & 0.40 & 3.10 \\
\hline 133 & & & & -31.55 & -30.15 & -29.25 & -28.45 & -27.30 & -2.65 & -0.40 & 1.00 & 2.25 & 3.35 \\
\hline 134 & 6.75 & 0.40 & 19.70 & -31.90 & -30.25 & -29.80 & -29.45 & -28.95 & -1.75 & 0.45 & 1.55 & 3.45 & 8.80 \\
\hline 135 & 3.45 & 0.15 & 27.10 & -31.80 & -31.69 & -31.05 & -29.61 & -27.70 & -2.20 & -1.28 & -0.50 & -0.12 & 0.10 \\
\hline 136 & 0.13 & 0.01 & 24.30 & -30.75 & -29.90 & -29.00 & -27.04 & -25.60 & -0.90 & -0.01 & 0.95 & 2.63 & 5.50 \\
\hline 137 & 2.26 & 0.13 & 20.70 & -31.95 & -31.30 & -30.50 & -29.27 & -28.10 & -1.30 & -0.39 & 0.16 & 0.67 & 1.45 \\
\hline 138 & 2.59 & 0.17 & 17.30 & -28.93 & -28.69 & -28.39 & -28.06 & -23.26 & -3.25 & -1.60 & -1.13 & -0.44 & 0.07 \\
\hline 139 & 1.53 & 0.13 & 13.60 & -30.42 & -29.46 & -28.59 & -27.50 & -27.25 & -3.97 & -3.03 & -2.27 & -0.92 & -0.30 \\
\hline 140 & 0.69 & 0.09 & 8.60 & -30.80 & -30.22 & -28.91 & -28.14 & -27.84 & -1.14 & 0.00 & 0.69 & 1.47 & 5.82 \\
\hline 141 & 0.52 & 0.12 & 5.18 & & & -29.92 & & & & & -3.70 & & \\
\hline 142 & 0.16 & 0.08 & 2.52 & -29.47 & -28.91 & -28.32 & -27.94 & -26.27 & -6.54 & -5.82 & -3.58 & -2.59 & -1.93 \\
\hline 143 & 3.38 & 0.12 & 32.64 & -33.76 & -33.15 & -32.55 & -31.92 & -31.87 & -1.80 & -1.07 & -0.85 & -0.35 & 0.72 \\
\hline 144 & 0.95 & 0.03 & 35.96 & -34.39 & -33.06 & -32.59 & -32.16 & -26.71 & -4.96 & -0.78 & 0.37 & 2.59 & 14.98 \\
\hline 145 & 0.04 & 0.00 & 10.60 & -26.93 & -26.53 & -25.67 & -24.82 & -24.42 & -2.44 & -2.35 & -2.16 & -1.97 & -1.88 \\
\hline 146 & 0.31 & 0.01 & 56.77 & -33.40 & -29.20 & -28.00 & -27.52 & -26.89 & -2.79 & -1.57 & -0.93 & -0.21 & 0.41 \\
\hline 147 & 0.02 & 0.01 & 3.12 & -26.57 & -26.32 & -25.40 & -25.19 & -25.15 & -2.59 & -2.28 & -1.94 & -1.58 & -0.84 \\
\hline 148 & 5.03 & 0.39 & 15.00 & -37.19 & -36.18 & -30.26 & -29.45 & -29.33 & -12.62 & -11.26 & -5.17 & -3.31 & -2.63 \\
\hline 149 & 6.35 & 0.19 & 38.39 & -37.50 & -36.49 & -36.17 & -34.56 & -27.95 & 0.42 & 1.42 & 1.61 & 1.88 & 2.57 \\
\hline 150 & 0.22 & 0.03 & 8.47 & -35.92 & -35.28 & -27.66 & -25.52 & -24.99 & -3.99 & -3.29 & 0.68 & 2.18 & 2.84 \\
\hline 151 & 1.55 & 0.07 & 27.30 & -35.74 & -29.42 & -29.03 & -28.72 & -28.11 & 0.89 & 1.89 & 2.49 & 2.87 & 3.23 \\
\hline 152 & 0.09 & 0.01 & 8.77 & -28.76 & -26.20 & -25.18 & -24.74 & -24.42 & 0.60 & 0.65 & 0.95 & 1.28 & 1.47 \\
\hline 153 & 1.00 & 0.06 & 20.77 & -33.68 & -33.16 & -32.21 & -28.52 & -27.37 & -2.26 & 0.40 & 1.14 & 2.37 & 2.72 \\
\hline
\end{tabular}


The $\delta^{15} \mathrm{~N}_{\text {sed }}$ data set exhibits pronounced secular variation (i.e., a range of $>10 \%$ ) and strong secular coherence (i.e., $74 \%$ of total variance is accounted for by the LOWESS curve). The secular coherence of the data set is significant in view of the relatively short residence time of nitrate in seawater ( 3 kyr) (Tyrrell, 1999; Brandes and Devol, 2002), which theoretically offers potential for strong $\delta^{15} \mathrm{~N}_{\mathrm{NO}_{3}^{-}}$variation at intermediate $\left(10^{3}-10^{6} \mathrm{yr}\right)$ timescales (Deutsch et al., 2004). Indeed, sub-Recent marine sediments exhibit a $\sim 14 \%$ range of $\delta^{15} \mathrm{~N}$ variation (Tesdal et al., 2012), reflecting local water mass effects linked to (1) strong $\mathrm{N}$ fixation, which can lower $\delta^{15} \mathrm{~N}_{\mathrm{NO}_{3}^{-}}$by several per mille, as in the Cariaco Basin and Baltic Sea, and (2) strong water-column denitrification, which can raise $\delta^{15} \mathrm{~N}_{\mathrm{NO}_{3}^{-}}$by $>10 \%$, as in upwelling systems in the Arabian Sea and the eastern tropical Pacific (Brandes and Devol, 2002; Gruber, 2008). However, the cumulative $\delta^{15} \mathrm{~N}$ distribution for sub-Recent sediments yields a mode of 5-6\%o with a standard deviation of $\pm 2.5 \%$ (Tesdal et al., 2012), which conforms well to the isotopic composition of modern seawater nitrate $(+4.8 \pm 0.2 \%$ o) (Sigman et al., 2000); note that the mean value of $6.7 \%$ reported by Tesdal et al. (2012) is skewed toward the high side by an overrepresentation of upwelling-zone sediments. Thus, the $\delta^{15} \mathrm{~N}_{\text {sed }}$ values of paleomarine units (Table 1) can be viewed as a random sample of a population of sediment $\delta^{15} \mathrm{~N}_{\text {sed }}$ values of a given age, the average of which is close to the $\delta^{15} \mathrm{~N}_{\mathrm{NO}_{3}^{-}}$of contemporaneous seawater. Although we cannot discount the possibility that some of our units are nonrepresentative of seawater $\delta^{15} \mathrm{~N}_{\mathrm{NO}_{3}^{-}}$of a given age, the broadly coherent pattern of secular variation recorded by our data set is not consistent with it being primarily a record of random local water mass effects (see Sect. 4.4).

\section{Discussion}

\subsection{The marine nitrogen cycle}

Long-term secular variation in $\delta^{15} \mathrm{~N}_{\text {sed }}$ and, by extension, in the $\delta^{15} \mathrm{~N}$ of seawater fixed nitrogen can be interpreted in terms of dominant processes of the marine $\mathrm{N}$ cycle, the main features of which are now well understood. Most bioavailable $\mathrm{N}$ is fixed by diazotrophic cyanobacteria with a fractionation of -1 to $-3 \%$ relative to the atmospheric $\mathrm{N}_{2}$ source $\left(\delta^{15} \mathrm{~N}_{\text {air }} \sim 0 \%\right.$ ) (Brandes and Devol, 2002; Gruber, 2008). Apart from assimilatory uptake, the major sinks for seawater fixed $\mathrm{N}$ are denitrification within the sediment or in the water column and the anammox process. Denitrification involves the bacterial use of nitrate as an oxidant in the respiration of organic matter with a maximum fractionation of $\sim-27 \%$ o (but commonly with an effective fractionation of $\sim-20 \pm 3 \%$ o), resulting in a strongly ${ }^{15} \mathrm{~N}$-enriched residual seawater nitrate pool. Denitrification in suboxic marine sediments typically yields much lower net fractionation $(\sim-1$ to $-3 \%$ ) owing to near-quantitative utilization of porewater nitrate (Sigman et al., 2003; Lehmann et al., 2004). The anammox reaction, in which ammonium and nitrate (or nitrite) are converted to $\mathrm{N}_{2}$, may eliminate more fixed $\mathrm{N}$ than denitrification in some marine environments (Kuypers et al., 2005), although the isotopic fractionation associated with this process is not well known (Galbraith et al., 2008).

The $\mathrm{N}$-isotopic composition of marine sediment depends on the $\delta^{15} \mathrm{~N}$ of seawater fixed $\mathrm{N}$, fractionation during assimilatory uptake, and subsequent alteration during decay in the water column and sediment (Robinson et al., 2012). Both ammonium and nitrate can be used as $\mathrm{N}$ sources in primary production, with fractionations of $-10( \pm 5) \%$ and $-3( \pm 2) \%$, respectively (Hoch et al., 1994; Waser et al., 1998). Nitrate is by far the more important source of $\mathrm{N}$ for eukaryotic marine algae, but ammonium is utilized by some modern microbial communities (Higgins et al., 2012) and may have been the main $\mathrm{N}$ substrate for eukaryotic algae during some oceanic anoxic events (OAEs; Altabet, 2001; Higgins et al., 2012). Assimilatory uptake enriches the residual fixed $\mathrm{N}$ pool in ${ }^{15} \mathrm{~N}$ and can result in shifts in the $\delta^{15} \mathrm{~N}_{\mathrm{NO} 3}$ - of local water masses (Hoch et al., 1994), but quantitative utilization of fixed $\mathrm{N}$ by marine autotrophs at annual timescales normally limits fractionation due to this process (Sigman et al., 2000; Somes et al., 2010). These processes determine the $\mathrm{N}$-isotopic composition of primary marine organic matter, before modification by diagenesis.

\subsection{Influence of diagenesis on sediment $\delta^{15} \mathrm{~N}$}

Diagenesis has the potential to alter the $\mathrm{N}$-isotopic composition of organic matter. First, selective degradation of amino acids can produce shifts of a few per mille in $\delta^{15} \mathrm{~N}_{\text {sed }}$ (Prahl et al., 1997; Gaye-Haake et al., 2005). Second, aerobic bacterial decomposition of organic matter results in deamination, i.e., the release of isotopically light $\mathrm{NH}_{4}^{+}$to sediment porewaters (Macko and Estep, 1984; Macko et al., 1987; Holmes et al., 1999), which results in ${ }^{15} \mathrm{~N}$ enrichment of the organic residue by a few per mille (Altabet, 1988; Libes and Deuser, 1988; François et al., 1992; Saino, 1992; Lourey et al., 2003). Subsequent nitrification can enrich the porewater $\mathrm{NH}_{4}^{+}$pool in ${ }^{15} \mathrm{~N}$ by $4-5 \%$, potentially leading to changes in bulk-sediment $\delta^{15} \mathrm{~N}_{\text {if }} \mathrm{NH}_{4}^{+}$diffuses back to the water column (Brandes and Devol, 1997; Prokopenko et al., 2006). However, if $\mathrm{NH}_{4}^{+}$generated within the sediment is captured by clay minerals, then the bulk sediment may show little or no change in $\delta^{15} \mathrm{~N}$ relative to the organic sinking flux (Higgins et al., 2012). Because decay processes can have variable effects on $\delta^{15} \mathrm{~N}_{\text {sed }}$, net fractionation can be either positive or negative relative to the unaltered source material. Surface sediments tend to be enriched in ${ }^{15} \mathrm{~N}$ by $1-5 \%$ relative to particulate organic nitrogen in the water column, possibly because the latter has undergone less extensive deamination (Brandes and Devol, 1997; Gaye-Haake et al., 2005; Prokopenko et al., 2006; Higgins et al., 2010). Differences 
between the $\delta^{15} \mathrm{~N}$ of the sinking and sediment fractions show water-depth dependence, reflecting greater oxic degradation of organic matter settling to the deep-ocean floor, although this effect is relatively small (Robinson et al., 2012). In contrast, rapid burial of organic matter in continental shelf and shelf-margin settings can yield sediment $\delta^{15} \mathrm{~N}$ values that are little modified from those of the organic export flux (Altabet and François, 1994; Altabet, 2001; Robinson et al., 2012).

Studies of $\mathrm{N}$ subfractions have been undertaken with the goal of recovering a $\mathrm{N}$-isotopic signature that is comparatively free of diagenetic effects. Chlorin $\mathrm{N}$ (Higgins et al., 2010) is ${ }^{15} \mathrm{~N}$-depleted relative to bulk-sediment $\mathrm{N}$ due to a $\sim 5 \%$ fractionation during photosynthesis (Sachs et al., 1999). Some studies have claimed large (up to $5 \%$ ) shifts in bulk-sediment $\delta^{15} \mathrm{~N}$ as a consequence of diagenesis (Sachs and Repeta, 1999). However, the studies of $\mathrm{N}$ subfractions cited above exhibit a systematic offset of 3-5\% between bulk-sediment and compound-specific $\delta^{15} \mathrm{~N}$ values that is consistent with the effects of photosynthetic fractionation overprinted by, at most, small $(<2 \%$ ) diagenetic effects. Following early diagenesis, deeper burial rarely causes more than minor changes in $\mathrm{N}$-isotopic compositions, as shown by (1) $\delta^{15} \mathrm{~N}$ variation of only a few per mille over a wide range of metamorphic grades (Imbus et al., 1992; Busigny et al., 2003; Jia and Kerrich, 2004), and (2) $\delta^{15} \mathrm{~N}$ values for metamorphosed units that are virtually indistinguishable from those of coeval unmetamorphosed units (e.g., compare the EoceneJurassic Franciscan Complex with age-equivalent units; Table 1). Ancient marine sediments are thus considered to be fairly robust recorders of the ambient isotopic composition of seawater fixed N (Altabet and François, 1994; Altabet et al., 1995; Higgins et al., 2010; Robinson et al., 2012).

\subsection{Influence of organic matter source on sediment $\delta^{15} \mathrm{~N}$}

All of the data used in this study represent bulk-sediment Nisotopic compositions, thus including both organic and inorganic nitrogen. The amount of mineral $\mathrm{N}$ present in most marine sediments is so small that it typically has little influence on bulk-sediment $\delta^{15} \mathrm{~N}$ (Holloway et al., 1998; Holloway and Dahlgren, 1999). In contrast, clay-adsorbed N (principally ammonium) can be quantitatively important, with concentrations of $\sim 0.1-0.2 \%$ in some marine units (e.g., Fig. 3 in Meyers, 1997; Fig. 3 in Lücke and Brauer, 2004; and Fig. S1 in Algeo et al., 2008). However, clay-adsorbed $\mathrm{N}$ is mostly derived from sedimentary organic matter, and the organic-toclay transfer of nitrogen is often at a late diagenetic stage, thus limiting translocation of $\mathrm{N}$ within the sediment column (Macko et al., 1986). These considerations suggest that the presence of a small inorganic $\mathrm{N}$ fraction in the study units is unlikely to affect our results.

In compiling the $\delta^{15} \mathrm{~N}_{\text {sed }}$ data set used in the present study, our principal concern was that admixture of large amounts of terrestrially sourced organic $\mathrm{N}$ might bias the marine $\delta^{15} \mathrm{~N}$ record. A number of different procedures can be used to

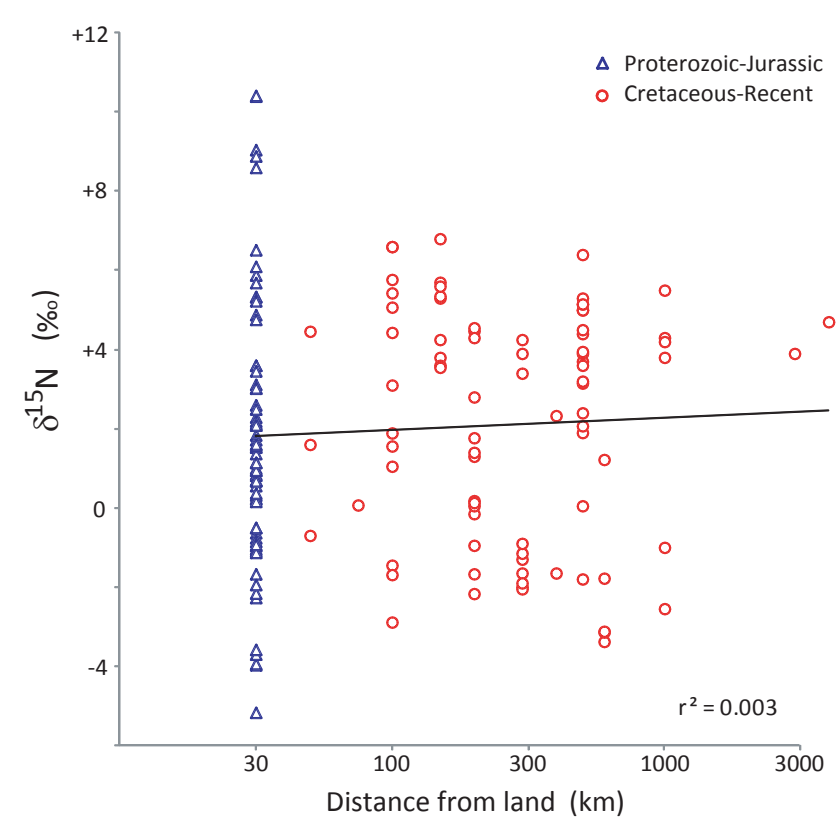

Fig. 2. Mean $\delta^{15} \mathrm{~N}_{\text {sed }}$ versus distance from land. All Proterozoic to Jurassic units were arbitrarily plotted at a distance of $30 \mathrm{~km}$ owing to their epicontinental settings and uncertainties regarding paleocoastal geography. Distances for Cretaceous to Recent units were measured from the paleogeographic map series of Ron Blakey (Colorado Plateau Geosystems, http://cpgeosystems.com/). Note the lack of any relationship between $\delta^{15} \mathrm{~N}$ and the distance of the depositional site from land.

screen samples for the presence of terrestrial organic matter, including petrographic analysis to identify maceral types (Hutton, 1987), biomarker analysis of steroids, polysaccharides, and hopane and tricyclic ratios (Huang and Meischein, 1979; Frimmel et al., 2004; Peters et al., 2004; Grice et al., 2005; Sephton et al., 2005; Wang and Visscher, 2007; Xie et al., 2007; Algeo et al., 2012), and hydrogen and oxygen indices (HI-OI) (Espitalié et al., 1977, 1985; Peters, 1986). Such proxies are generally reliable in distinguishing organic matter sources, subject to some caveats (Meyers et al., 2009a). These types of proxies were available only for a subset of the present study units (Table 1), but, where available, they generally confirmed the dominance of marine over terrestrial organic matter. Studies of modern continental shelf sediments show a rapid decline in the proportion of terrestrial organic matter away from coastlines (Hedges et al., 1997; Hartnett et al., 1998). The study units of Proterozoic to Jurassic age were mostly epicontinental and, hence, deposited close to land areas (Fig. 2), although there was little terrestrial vegetation for export to marine systems prior to the Devonian (Kenrick and Crane, 1997). In contrast, the study units of Cretaceous to Recent age (which are mostly from Deep Sea Drilling Project (DSDP), Ocean Drilling Program (ODP), and Integrated Ocean Drilling Program (IODP) cores) overwhelmingly represent open-ocean 


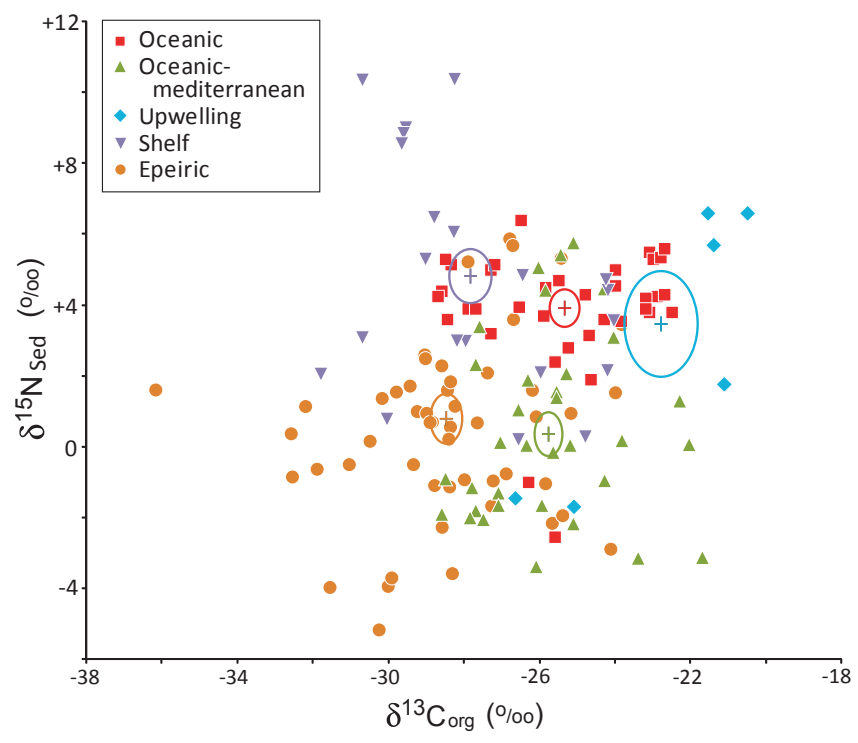

Fig. 3. $\delta^{15} \mathrm{~N}_{\text {sed }}$ versus $\delta^{13} \mathrm{C}_{\text {org }}$ for 153 marine study units. The average $\delta^{15} \mathrm{~N}_{\text {sed }}-\delta^{13} \mathrm{C}_{\text {org }}$ compositions for five depositional settings are shown by crosses (mean) and ovals (one standard error of the mean). The data set as a whole exhibits no significant covariation between $\delta^{15} \mathrm{~N}_{\text {sed }}$ and $\delta^{13} \mathrm{C}_{\text {org }}$. However, there are statistically significant differences in average $\delta^{15} \mathrm{~N}_{\text {sed }}-\delta^{13} \mathrm{C}_{\text {org }}$ compositions by depositional setting (see Sect. 4.4 and Fig. 5).

and distal continent-margin sites that were at a significant remove from land areas (Fig. 2) and, hence, unlikely to have accumulated large amounts of terrestrial organic matter.

Sediment $\mathrm{C}_{\mathrm{org}}: \mathrm{N}$ ratios potentially also provide insights regarding organic matter sources (Meyers, 1994, 1997). Terrestrial organic matter is characterized by high $\mathrm{C}_{\text {org }}: \mathrm{N}$ ratios ( 20-200) owing to an abundance of N-poor cellulose in land plants (Ertel and Hedges, 1985). In contrast, fresh marine organic matter exhibits low $\mathrm{C}_{\text {org }}$ : $\mathrm{N}$ ratios $(\sim 4-10)$ owing to a lack of cellulose and an abundance of N-rich proteins in planktic algae (Müller, 1977). Diagenesis can result in either lower $\mathrm{C}_{\text {org }}: \mathrm{N}$ ratios through preferential preservation of organic $\mathrm{N}$ as clay-adsorbed ammonium, or higher $\mathrm{C}_{\text {org }}: \mathrm{N}$ ratios through preferential loss of proteinaceous components (Meyers, 1994). Covariation between $\delta^{15} \mathrm{~N}, \delta^{13} \mathrm{C}_{\mathrm{org}}$, and $\mathrm{C}_{\text {org }}: \mathrm{N}$ ratios can reveal mixing relationships in estuarine (Thornton and McManus, 1994; Ogrinc et al., 2005) and marine sediments (Müller, 1977; Meyers et al., 2009b). In our Phanerozoic data set, $\delta^{13} \mathrm{C}_{\text {org }}$ and $\delta^{15} \mathrm{~N}$ exhibit no relationship $\left(r^{2}=0.01\right.$; Fig. 3$)$, but $\delta^{15} \mathrm{~N}$ exhibits moderate negative covariation with $\mathrm{C}_{\mathrm{org}}: \mathrm{N}\left(r^{2}=0.21 ; p(\alpha)<0.001\right.$; Fig. 4). The source of the latter relationship is uncertain. Although conceivably representing a marine-terrestrial mixing trend, this interpretation is unlikely given that the majority of units with low $\delta^{15} \mathrm{~N}$ and high $\mathrm{C}_{\mathrm{org}}: \mathrm{N}$ values come from openmarine settings of Cretaceous-Recent age that presumably contain little terrestrial organic matter. The linkage of higher $\mathrm{C}_{\text {org }}: \mathrm{N}$ ratios (to $\sim 40$ ) with lower $\delta^{15} \mathrm{~N}$ values is particu-

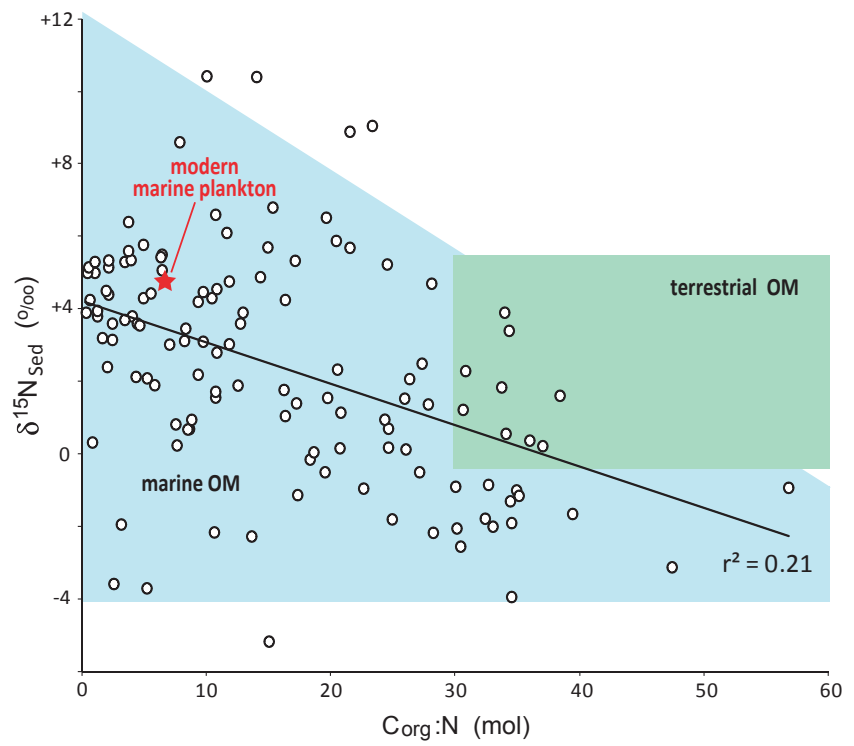

Fig. 4. $\delta^{15} \mathrm{~N}_{\text {sed }}$ versus $\mathrm{C}_{\text {org }}$ : $\mathrm{N}$ ratio. Average composition of modern marine plankton shown by red star, and approximate compositional range of terrestrial (i.e., soil-derived) organic matter by green rectangle. Note that the pattern of negative covariation between $\delta^{15} \mathrm{~N}_{\text {sed }}$ and $\mathrm{C}_{\text {org }}: \mathrm{N}$ is not clearly associated with a terrestrial endmember and does not provide evidence of pervasive mixing of marine and terrestrial organic matter in our study units.

larly characteristic of organic-rich sediments deposited under anoxic conditions (e.g., Junium and Arthur, 2007). This pattern has been attributed to enhanced cyanobacterial $\mathrm{N}$ fixation under N-poor conditions in restricted anoxic marine basins (Junium and Arthur, 2007) but potentially might be due to enhanced assimilatory recycling of ${ }^{15} \mathrm{~N}$-depleted ammonium in such settings (Higgins et al., 2012).

The relatively N-poor nature of terrestrial organic matter means that, even if present in modest quantities, it is unlikely to have had much influence on bulk sediment $\delta^{15} \mathrm{~N}$. For example, in a 50:50 mixture of marine and terrestrial organic matter, $\sim 80-95 \%$ of total $\mathrm{N}$ will be of marine origin because of the lower $\mathrm{C}_{\text {org }}: \mathrm{N}$ ratios of marine organic matter $(\sim 4-10)$ relative to terrestrial organic matter $(\sim 20-200)$ (Meyers, 1994, 1997). Where mixing proportions have been quantified, the terrestrial organic fraction is more commonly in the range of 10-20\% (e.g., Jaminski et al., 1998; Algeo et al., 2008), in which case $>95 \%$ of total $\mathrm{N}$ is marinederived. Although we cannot conclusively demonstrate that our Phanerozoic marine $\delta^{15} \mathrm{~N}$ trend (Fig. 1) is uninfluenced by terrestrial contamination, we infer that such influences were probably minimal, and that the observed pattern of secular variation in $\delta^{15} \mathrm{~N}_{\text {sed }}$ broadly reflects the isotopic composition of contemporaneous seawater fixed $\mathrm{N}$. 


\subsection{Influence of depositional setting on sediment $\delta^{15} \mathrm{~N}$}

One important issue is whether our $\delta^{15} \mathrm{~N}_{\text {sed }}$ data set records variation in a global parameter (i.e., seawater nitrate $\delta^{15} \mathrm{~N}$ ) or represents mainly local water mass effects in which sediment $\delta^{15} \mathrm{~N}$ varied as a function of depositional setting. Owing to unevenness in the distribution of depositional settings in our data set through time, we cannot answer this question definitively, but the following analysis provides some insight as to the relative importance of local versus global controls on sediment $\delta^{15} \mathrm{~N}$.

We classified the 153 study units into five categories of depositional setting: (1) oceanic, i.e., unrestricted deep marine; (2) oceanic-mediterranean, i.e., restricted deep marine; (3) upwelling, i.e., open continental margin/slope with a known upwelling system; (4) shelf, i.e., open continental margin without upwelling; and (5) epeiric sea, i.e., a cratonic-interior shelf or basin. When viewed as a function of time (Fig. 5), it is apparent that there is a major change in depositional settings in the mid-Mesozoic: all pre-Cretaceous units are from either shelf or epeiric-sea settings, whereas Cretaceous to Recent units are mostly oceanic or oceanic-mediterranean with a small number from other settings. The reason for this shift is that nearly all pre-Cretaceous units were collected in outcrop and represent cratonic deposits, whereas the majority of Cretaceous and younger units were collected during deepsea (DSDP, ODP, or IODP) cruises and represent deep-ocean deposits. Thus, the ultimate control on the age distribution of depositional settings in our data set is the age distribution of present-day oceanic crust.

Several significant observations can be gleaned from the age distribution of depositional settings (Fig. 5). First, relatively young (i.e., Neogene) oceanic units exhibit an average $\delta^{15} \mathrm{~N}_{\text {sed }}(+4.2 \pm 0.8 \%$ o $)$ that overlaps with and is only marginally depleted relative to the $\mathrm{N}$-isotopic composition of present-day seawater nitrate $(+4.8-5.0 \%$ ) (Sigman et al., 2000). This observation is consistent with the inference that $\delta^{15} \mathrm{~N}_{\text {sed }}$ is a relatively robust recorder of seawater $\delta^{15} \mathrm{~N}_{\mathrm{NO}_{3}^{-}}$(Altabet and François, 1994; Altabet et al., 1995; Higgins et al., 2010; Robinson et al., 2012). Second, the range of $\delta^{15} \mathrm{~N}_{\text {sed }}$ variation shown by Neogene units as a function of depositional setting is limited: on average, upwelling units $(+5.5 \pm 2.1 \%$ o are just $1.3 \%$ enriched and oceanicmediterranean units $(+1.2 \pm 2.2 \%$ ) just $3.0 \%$ depleted in ${ }^{15} \mathrm{~N}$ relative to oceanic units (Fig. 3). While these differences are statistically significant (at $p(\alpha)<0.01$ ), they are much smaller than the $>10 \%$ range of $\delta^{15} \mathrm{~N}_{\text {sed }}$ variation observed through the Phanerozoic (Fig. 1). Third, secular variation in $\delta^{15} \mathrm{~N}_{\text {sed }}$ is coherent across the mid-Mesozoic "junction" at which pre-Cretaceous epeiric/shelf units yield to Cretaceous and younger oceanic/oceanic-mediterranean units (Fig. 5). This observation is significant because it suggests that different kinds of depositional settings are recording a common signal that shows up in both cratonic interiors and the deep ocean. While local influences are likely to have modified the

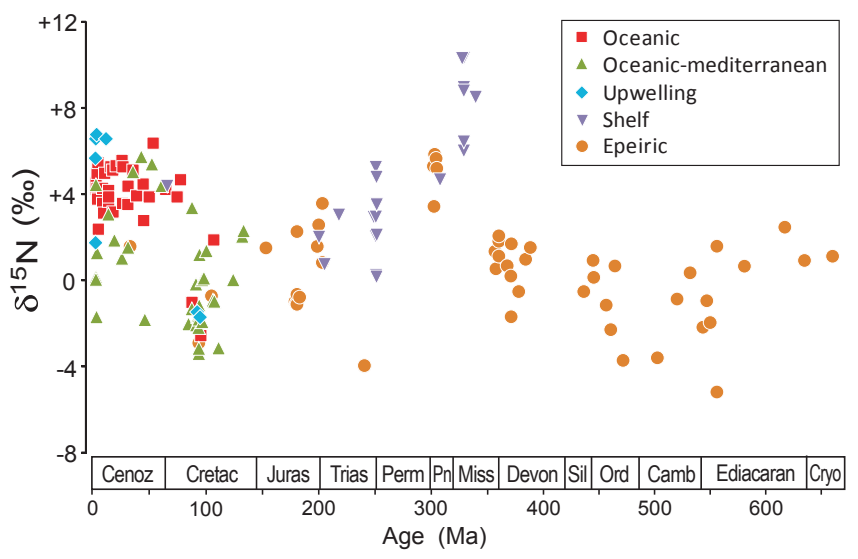

Fig. 5. $\delta^{15} \mathrm{~N}_{\text {sed }}$ as a function of depositional setting. For the Neogene, upwelling units are $1.3 \%$ enriched and oceanicmediterranean units $3.0 \%$ depleted in ${ }^{15} \mathrm{~N}$ on average relative to oceanic units. For units of any given age, note the generally limited variation in $\delta^{15} \mathrm{~N}_{\text {sed }}$ among different setting types, relative to the larger variation in $\delta^{15} \mathrm{~N}_{\text {sed }}$ through time. For the Phanerozoic as a whole, note the smooth $\delta^{15} \mathrm{~N}_{\text {sed }}$ transition in the midMesozoic between entirely different setting types, i.e., epeiric and shelf in the Jurassic and earlier versus mainly oceanic and oceanicmediterranean in the Cretaceous and later.

$\mathrm{N}$-isotopic composition of some study units, the foregoing observations are not consistent with the hypothesis that our Phanerozoic $\delta^{15} \mathrm{~N}_{\text {sed }}$ record is dominated by such influences. We infer that there is a dominant underlying secular signal present in the $\delta^{15} \mathrm{~N}_{\text {sed }}$ data set that is independent of setting type and that reflects a global control, i.e., seawater $\delta^{15} \mathrm{~N}_{\mathrm{NO}_{3}^{-}}$.

In our data set, most of the Phanerozoic is characterized by a relatively low density of data (averaging one data point per $4-5$ million years). However, a few narrow $(\leq 1-\mathrm{Myr}-$ long) time slices are represented by multiple data points, providing a basis for assessing spatial variance at certain times in the past. One such interval is the Cenomanian-Turonian boundary (Fig. 6). During this interval, the range of variation in unit-mean $\delta^{15} \mathrm{~N}_{\text {sed }}$ values is just $1.7 \%$ (i.e., -2.9 to $-1.2 \%$ o) for sites ranging from high northern to high southern paleolatitudes. While the majority of these units represent oceanic-mediterranean settings in the young North Atlantic and South Atlantic basins, similar $\delta^{15} \mathrm{~N}_{\text {sed }}$ values are nonetheless observed in epeiric ( $-2.9 \%$ in England) and upwelling settings ( $-1.6 \%$ in Morocco) (Jenkyns et al., 2007) as well as outside the Atlantic region $(-2.6 \%$ on the Kerguelen Plateau) (Meyers et al., 2009a). Thus, these data imply a relatively uniform $\mathrm{N}$-isotopic composition for global seawater nitrate at the Cenomanian-Turonian boundary. Further, almost all of these regions exhibit a +4 to $+5 \%$ o shift in $\delta^{15} \mathrm{~N}_{\text {sed }}$ for units of latest Cretaceous to early Paleogene age (Fig. 1; Table 1), consistent with our hypothesis of a global shift in seawater $\delta^{15} \mathrm{~N}_{\mathrm{NO}_{3}^{-}}$during the Late Cretaceous. 


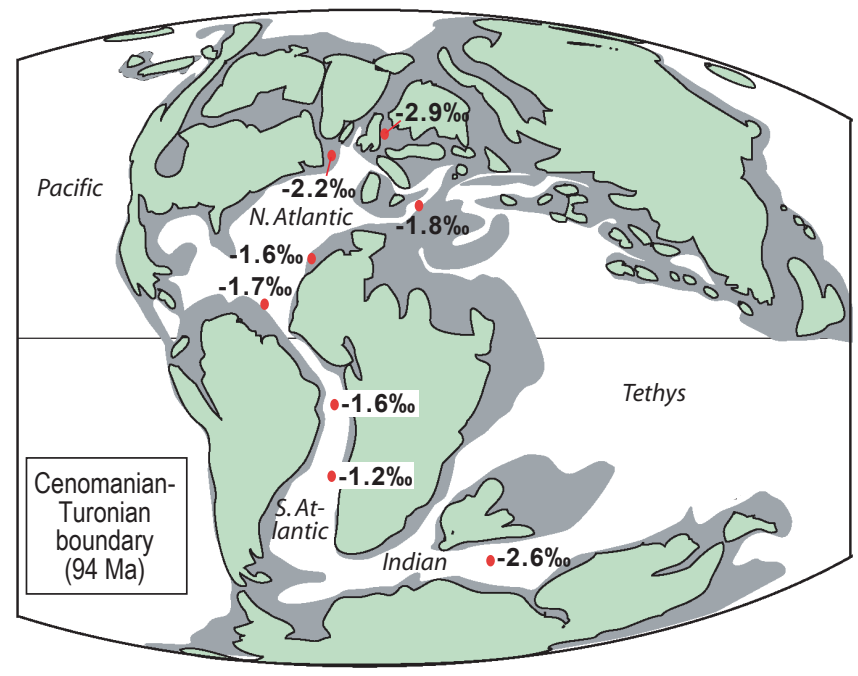

Fig. 6. Average $\delta^{15} \mathrm{~N}_{\text {sed }}$ for Cenomanian-Turonian boundary units. Note the limited range of $\delta^{15} \mathrm{~N}_{\text {sed }}$ values $(-2.9 \%$ o to $-1.2 \%$ ) for sites ranging from high northern to high southern paleolatitudes, implying a relatively uniform contemporaneous seawater $\delta^{15} \mathrm{~N}_{\mathrm{NO}_{3}^{-}}$ composition. Data sources in Table 1.

Another time slice with multiple data points is the Permian-Triassic boundary (PTB; Fig. 7). The range of $\delta^{15} \mathrm{~N}_{\text {sed }}$ variation observed at the PTB is somewhat greater than for the Cenomanian-Turonian boundary, but the geographic distribution of units is wider and their setting types are more diverse as well. Late Permian units predating the PTB crisis exhibit a $\delta^{15} \mathrm{~N}_{\text {sed }}$ range of $4.6 \%$ (i.e., $+0.3 \%$ to $+4.9 \%$ ) but show spatially coherent variation: low values characterize the central Panthalassic Ocean $(+0.3 \%$ ), intermediate values the Tethyan region (mostly +2.0 to $+3.6 \%$ ), and high values the northwestern Pangean margin $(+3.5$ to $+4.9 \%$ ). This pattern is likely to reflect regional variation in the intensity of water-column denitrification, which was higher in the high-productivity oceanic cul-de-sac formed by the Tethys Ocean (Mii et al., 2001; Grossman et al., 2008) and in the northwest Pangean upwelling system (Beauchamp and Baud, 2002; Schoepfer et al., 2012, 2013). Despite major changes in seawater temperature and dissolved oxygen levels in conjunction with the PTB crisis (Romano et al., 2012; Sun et al., 2012; Song et al., 2013), marine units show remarkably little change in $\delta^{15} \mathrm{~N}$ across the PTB: Lower Triassic unit means range from $-0.4 \%$ o to $+5.3 \%$, and the magnitude of the PTB shift at individual locales varies from $-2.8 \%$ o to $+0.4 \%$ o with an average of $-0.9 \%$. Negative $\delta^{15} \mathrm{~N}_{\text {sed }}$ shifts at the PTB have been attributed to enhanced $\mathrm{N}$ fixation rates (Luo et al., 2011). However, these shifts are consistent with our hypothesis of lowered seawater $\delta^{15} \mathrm{~N}_{\mathrm{NO} 3}$ values as a consequence of enhanced rates of sedimentary (relative to water-column) denitrification during greenhouse climate intervals such as that of the Early Triassic (Romano et al., 2012; Sun et al., 2012).

\subsection{Marine nitrogen cycle modeling}

We employed a reservoir box model to investigate possible controls on long-term secular variation in seawater $\delta^{15} \mathrm{~N}_{\mathrm{NO}_{3}^{-}}$ (see Appendix $\mathrm{C}$ for model details). Seawater $\delta^{15} \mathrm{~N}_{\mathrm{NO}_{3}^{-}}$can be approximated from a steady-state isotope mass balance that assumes $\mathrm{N}$ fixation $\left(f_{\mathrm{FIX}}\right)$ as the primary source and sedimentary $\left(f_{\mathrm{DS}}\right)$ and water-column $\left(f_{\mathrm{DW}}\right)$ denitrification as the two largest sinks for seawater fixed $\mathrm{N}$ (Brandes and Devol, 2002; Deutsch et al., 2004; Gruber, 2008). We assumed that the marine $\mathrm{N}$ cycle is in a homeostatic steadystate condition at geologic timescales (DeVries et al., 2013), and thus that losses of fixed $\mathrm{N}$ to denitrification are balanced by new $\mathrm{N}$ fixation (i.e., $f_{\mathrm{DW}}+f_{\mathrm{DS}}=f_{\mathrm{FIX}}$ ), which is consistent with the strong spatial coupling of these processes in the modern ocean (Galbraith et al., 2004; Deutsch et al., 2007; Knapp et al., 2008). Our baseline scenario utilized fluxes and fractionation factors based on the modern marine $\mathrm{N}$ cycle - i.e., $f_{\mathrm{FIX}}=220 \mathrm{Tg} \mathrm{a}^{-1}, f_{\mathrm{DS}}=160 \mathrm{Tg} \mathrm{a}^{-1}, f_{\mathrm{DW}}=$ $60 \mathrm{Tg} \mathrm{a}^{-1}, \varepsilon_{\mathrm{FIX}}=-2 \% o, \varepsilon_{\mathrm{DS}}=-2 \%$, and $\varepsilon_{\mathrm{DW}}=-20 \%$ o - where $\varepsilon$ represents the fractionations associated with $\mathrm{N}$ source and sink fluxes $(f)$, and the photosynthetic fractionation factor $\left(\varepsilon_{\mathrm{P}}\right)$ linked to nitrate utilization is $0 \%$. This scenario yields an equilibrium seawater $\delta^{15} \mathrm{~N}_{\mathrm{NO}_{3}^{-}}$of $+4.9 \%$ that matches the composition of fixed $\mathrm{N}$ in the present-day deep ocean (Sigman et al., 2000).

The most important influence on global seawater $\delta^{15} \mathrm{~N}_{\mathrm{NO}_{3}^{-}}$ variation in our model is the fraction of denitrification that occurs in the water column ( $F_{\mathrm{DW}}$, calculated as $f_{\mathrm{DW}} /\left(f_{\mathrm{DW}}+\right.$ $\left.f_{\mathrm{DS}}\right)$; Fig. 8). In our baseline scenario ( $\varepsilon_{\mathrm{DW}}=-20 \%$ o, $\varepsilon_{\mathrm{P}}=$ $0 \%$ ), the modern seawater $\delta^{15} \mathrm{~N}_{\mathrm{NO}_{3}^{-}}$of $\sim+4.9 \%$ corresponds to $F_{\mathrm{DW}}$ of 0.27 (point 1, Fig. 8b), which is close to recent estimates of 0.29 (DeVries et al., 2012) and 0.36 (Eugster and Gruber, 2012). However, the same $\delta^{15} \mathrm{~N}_{\mathrm{NO}_{3}^{-}}$composition can be achieved with other model parameterizations. Laboratory culture studies indicate that $\varepsilon_{\mathrm{DW}}$ might be as low as $-10 \%$ in some marine systems (Kritee et al., 2012). Reducing $\varepsilon_{\text {DW }}$ to $-15 \% \circ$ and $-10 \%$ yields $F_{\text {DW }}$ of 0.37 and 0.62 (points 2 and 3, Fig. 8b); the former is still within the range of $F_{\mathrm{DW}}$ estimates for modern marine systems (cf. Eugster and Gruber, 2012) although the latter is not. Our baseline scenario assumes no net fractionation linked to photosynthetic assimilation of seawater nitrate $\left(\varepsilon_{\mathrm{P}}=0 \%\right.$ ) (Brandes and Devol, 2002; Gruber, 2008; Granger et al., 2010), but uptake of ammonium is accompanied by a significant negative fractionation (Hoch et al., 1994; Waser et al., 1998), and a recent study lends support to the hypothesis that recycled ammonium was a major source of fixed $\mathrm{N}$ for eukaryotic algae during some OAEs (Higgins et al., 2012). We modeled the effects of variable photosynthetic fractionation with $\varepsilon_{\mathrm{P}}$ values of $-4 \%$ and $-8 \%$, which yield $\delta^{15} \mathrm{~N}_{\mathrm{NO}_{3}^{-}}$equal to $+4.9 \%$ when $F_{\text {DW }}$ is 0.48 and 0.72 , respectively (points 4 and 5 , Fig. $8 \mathrm{~b}$ ). These $F_{\mathrm{DW}}$ values are improbably large for the modern (icehouse) marine $\mathrm{N}$ cycle, but nonzero values of 


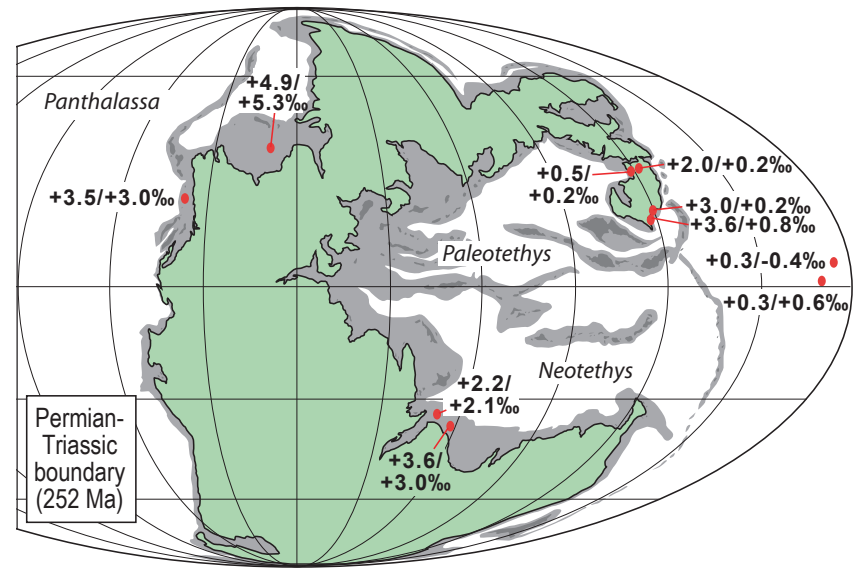

Fig. 7. Average $\delta^{15} \mathrm{~N}_{\text {sed }}$ for Upper Permian (left) and Lower Triassic (right) units. Upper Permian $\delta^{15} \mathrm{~N}_{\text {sed }}$ varies from low values in the central Panthalassic Ocean $(+0.3 \%$ ) to intermediate values in the Tethyan region and high values on the northwestern Pangean margin $(+4.9 \%$ o), probably owing to higher rates of water-column denitrification in the latter regions. Note that all Permo-Triassic $\delta^{15} \mathrm{~N}_{\text {sed }}$ values globally are intermediate relative to the low values characteristic of the Cenomanian-Turonian boundary or the high values characteristic of the Carboniferous (Fig. 1). Site-specific changes in $\delta^{15} \mathrm{~N}_{\text {sed }}$ across the PTB range from $-2.8 \%$ o to $+0.4 \%$ o with an average of $-0.9 \%$, which is consistent with our hypothesis of intensified sedimentary denitrification during greenhouse climate intervals such as the Early Triassic. Data sources in Table 1 with additional data from S. Schoepfer and T. Algeo (unpubl. data).

$\varepsilon_{\mathrm{P}}$ may have been important during greenhouse intervals (see below).

The variations in seawater $\delta^{15} \mathrm{~N}_{\mathrm{NO}_{3}^{-}}$between icehouse and greenhouse climate modes observed in our long-term $\delta^{15} \mathrm{~N}_{\text {sed }}$ record (Fig. 1) are an indication of major secular changes in the marine $\mathrm{N}$ cycle. In our baseline scenario, the peak icehouse $\delta^{15} \mathrm{~N}_{\mathrm{NO}_{3}^{-}}$of $\sim+8 \%$ yields $F_{\mathrm{DW}}$ of $\sim 0.45$ (point 6, Fig. 8b), indicating an increase in water-column denitrification relative to the modern ocean. Although the same $\delta^{15} \mathrm{~N}_{\mathrm{NO}_{3}^{-}}$can be achieved with $\varepsilon_{\text {DW }}$ of $-15 \%$ and $-10 \%$, the resulting $F_{\mathrm{DW}}$ values $(0.62$ and 0.98 ; points 7 and 8 , Fig. 8b) are improbably large. Lack of evidence for ammonium recycling during icehouse modes makes nonzero $\varepsilon_{\mathrm{P}}$ values unlikely, which in any case would yield equally improbable values of $F_{\mathrm{DW}}$. The minimum greenhouse $\delta^{15} \mathrm{~N}_{\mathrm{NO}_{3}^{-}}$ of $\sim-3 \%$ cannot be achieved in our baseline scenario even when $F_{\mathrm{DW}}$ is reduced to 0 (point 9, Fig. 8b). However, evidence for strong ammonium recycling in greenhouse oceans (Higgins et al., 2012) indicates that $\varepsilon_{\mathrm{P}}$ may have been nonzero at those times. Decreasing $\varepsilon_{\mathrm{P}}$ to $-4 \%$ and $-8 \%$ o yields $F_{\text {DW }}$ of 0.10 and 0.33 (points 10 and 11 , Fig. $8 \mathrm{~b}$ ), the former representing a large decrease in $F_{\mathrm{DW}}$ relative to the modern ocean. Since $\varepsilon_{\mathrm{P}}$ of $-8 \%$ represents an absolute minimum (i.e., recycling of nearly all seawater $\mathrm{N}$ as ammonium), $\varepsilon_{\mathrm{P}}=-4 \%$ is a more reasonable estimate for anoxic marine systems with mixed utilization of recycled ammonium and cyanobacterially fixed $\mathrm{N}$ (Higgins et al., 2012). Note that, at low $F_{\mathrm{DW}}$, variation in $\varepsilon_{\mathrm{DW}}$ has little effect on $\delta^{15} \mathrm{~N}_{\mathrm{NO}_{3}^{-}}$. In summary, the most likely scenario to account for long-term secular shifts in $\delta^{15} \mathrm{~N}_{\mathrm{NO}_{3}^{-}}$(Fig. 1) within existing $\mathrm{N}$-budget and $\mathrm{N}$-isotopic constraints is for (1) $F_{\mathrm{DW}}$ to vary between $\sim 0.2$ and 0.5 (permissive of $\varepsilon_{\mathrm{DW}}$ values between -15 and $-20 \%$ ) with $\varepsilon_{\mathrm{P}}=0 \%$ during icehouse climate modes, and (2) $F_{\text {DW }}$ to decrease to $\sim 0.1-0.2$ with a shift in $\varepsilon_{\mathrm{P}}$ to ca. $-4 \%$ during greenhouse climate modes.

\subsection{Controls on long-term variation in the marine nitrogen cycle}

Although enhanced water-column denitrification has been inferred during the warm climate intervals that produced OAEs (Rau et al., 1987; Jenkyns et al., 2001; Junium and Arthur, 2007), strong ${ }^{15} \mathrm{~N}$ depletion of contemporaneous sediments is inconsistent with globally elevated water-column denitrification rates. Our inference of reduced water-column denitrification during greenhouse climate modes (Fig. 9a) contradicts the existing paradigm linking OAEs to high watercolumn denitrification rates (Rau et al., 1987; Jenkyns et al., 2001; Junium and Arthur, 2007). A reconciliation of these views is possible if rates were high regionally in semirestricted marine basins such as the proto-South Atlantic but reduced on a globally integrated basis. Our results are also at odds with the observation that modern upwelling zones exhibit peak $\delta^{15} \mathrm{~N}_{\text {sed }}$ values in conjunction with deglaciations rather than glacial maxima (François et al., 1992; Altabet et al., 1995; Ganeshram et al., 1995). While the latter relationship is valid at intermediate timescales, our results indicate that $F_{\mathrm{DW}}$ is higher on a time-averaged basis (i.e., integrating glacial-interglacial variation) for icehouse modes than for greenhouse modes. This inference is supported by a study of Plio-Pleistocene sediments in the eastern tropical Pacific, in which $\delta^{15} \mathrm{~N}_{\text {sed }}$ rose by $\sim 2 \%$ o following a cooling event at 2.1 Ma (Liu et al., 2008), consistent with an increase in time-averaged water-column denitrification rates. We infer that transient, albeit repeated, shifts in favor of watercolumn denitrification (i.e., higher $F_{\mathrm{DW}}$ ) during the interglacial stages of icehouse climate intervals have resulted in a sustained (i.e., multimillion-year) shift toward higher seawater $\delta^{15} \mathrm{~N}_{\mathrm{NO}_{3}^{-}}$that has been captured by the long-term $\delta^{15} \mathrm{~N}_{\text {sed }}$ record (Fig. 1). Such a long-term climate-related shift in seawater $\delta^{15} \mathrm{~N}_{\mathrm{NO}_{3}^{-}}$can occur if the positive shift associated with each glacial epoch is larger than the negative shift associated with each interglacial epoch, resulting in progressively more ${ }^{15} \mathrm{~N}$-enriched compositions for icehouse intervals relative to greenhouse intervals.

Several mechanisms might potentially link variations in seawater $\delta^{15} \mathrm{~N}_{\mathrm{NO}_{3}^{-}}$to long-term climate cycles. Sea-level elevation is known to influence the locus of denitrification in marine systems (Deutsch et al., 2004). High sea-level 

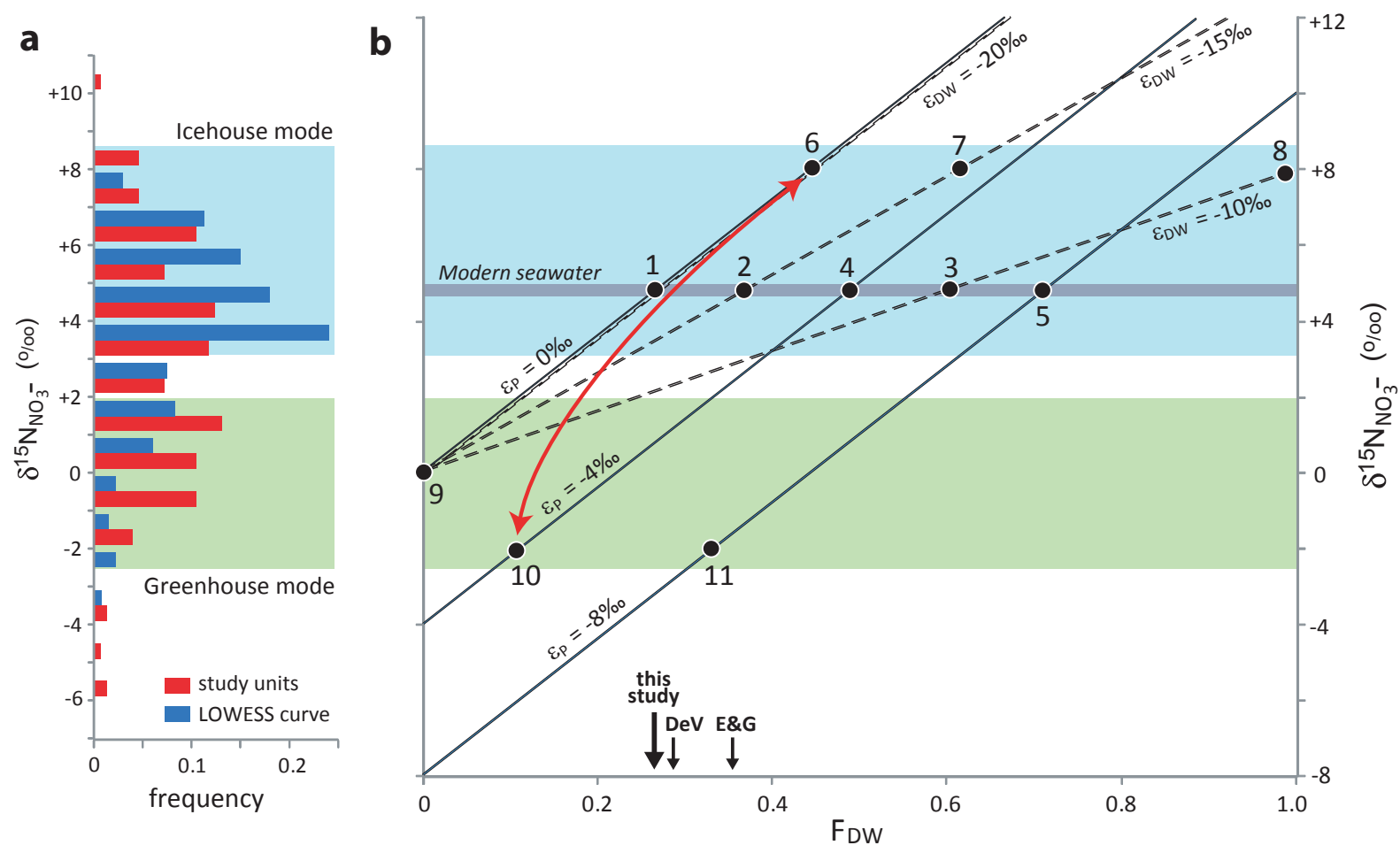

Fig. 8. $\delta^{15} \mathrm{~N}$ distributions and modeling constraints. (a) Frequency distributions for unit-median $\delta^{15} \mathrm{~N}_{\text {sed }}$ values (red) and LOWESS curve values (blue). Note that both distributions exhibit bimodal character, with modes at $\sim-2$ to $+2 \%$ and $\sim+4$ to $+8 \%$ representative of greenhouse and icehouse climate modes, respectively. (b) Reservoir box model estimates of $\delta^{15} \mathrm{~N}_{\mathrm{NO}_{3}^{-}}$as a function of the fraction of watercolumn denitrification $\left(F_{\mathrm{DW}}\right)$. The dashed diagonal lines represent variable fractionation during water-column denitrification $\left(\varepsilon_{\mathrm{DW}}\right)$, and the solid diagonal lines variable fractionation during photosynthetic uptake of seawater fixed $\mathrm{N}(\varepsilon \mathrm{P})$. Colored fields show the isotopic range of marine $\delta^{15} \mathrm{~N}_{\text {sed }}$ during greenhouse (green) and icehouse (blue) climate modes as well as modern seawater $\delta^{15} \mathrm{~N}_{\mathrm{NO}_{3}^{-}}($gray) (Sigman et al., 2000). Arrows at bottom show $F_{\mathrm{DW}}$ of 0.27 (this study), 0.29 (DeVries et al., 2012), and 0.36 (Eugster and Gruber, 2012). The red curve represents our "most likely scenario" of concurrent changes in $F_{\mathrm{DW}}$ and $\varepsilon_{\mathrm{P}}$ as a function of greenhouse-icehouse climate shifts. See text for discussion of numbered points.

elevations during greenhouse climate modes favor sedimentary denitrification owing to greater burial of organic matter on continental shelves (Fig. 9a), whereas low sealevel elevations during icehouse climate modes favor watercolumn denitrification through elevated organic carbon sinking fluxes to the thermocline and expansion of oceanic oxygen-minimum zones (Fig. 9b). A first-order sea-level control on the marine $\mathrm{N}$ cycle is consistent with existing records of Phanerozoic eustasy and continental flooding (Fig. 10). Eustasy shows a strong relationship to firstorder climate modes, with long-term rises or highstands during greenhouse intervals and long-term falls or lowstands during icehouse intervals. $\delta^{15} \mathrm{~N}_{\text {sed }}$ exhibits a distinct pattern of negative covariation with eustatic elevation for the Phanerozoic as a whole $\left(r^{2}=0.18\right.$; Fig. 11). This relationship is even stronger for Cretaceous-Recent units alone $\left(r^{2}=0.37\right)$, probably because both the $\delta^{15} \mathrm{~N}_{\text {sed }}$ and eustatic records are more securely defined for this interval than for the pre-Cretaceous. $\delta^{15} \mathrm{~N}_{\text {sed }}$ also exhibits negative covariation with long-term continental flooding records (Fig. 10), although the relationship is not as strong as for eustasy due to several factors: (1) greater vintage of the flooding records, (2) provinciality of the Sloss (1963) record (which represents only North America), and (3) low resolution of the Ronov (1984) record (which provides only 2-3 area estimates for most geologic periods).

A second potential mechanism linking long-term variation in the marine $\mathrm{N}$ cycle to first-order Phanerozoic climate cycles may be through tectonic controls. In this scenario, changes in oceanic gateways and circulation patterns can alter the locus of denitrification through changes in upwelling intensity or thermocline ventilation. In our long-term $\delta^{15} \mathrm{~N}_{\text {sed }}$ record, the mid-Early Mississippian and mid-Late Cretaceous feature as intervals of potentially rapid changes in seawater $\delta^{15} \mathrm{~N}_{\mathrm{NO}_{3}^{-}}$(Fig. 1). Both of these $\mathrm{N}$-isotopic shifts occur in the "transitional interval" between greenhouse and icehouse climate modes and, thus, tens of millions of years in advance of peak glaciation and eustatic lowstand. On the other hand, these $\mathrm{N}$-isotopic shifts coincided with major tectonic events that are likely to have altered global-ocean 
a

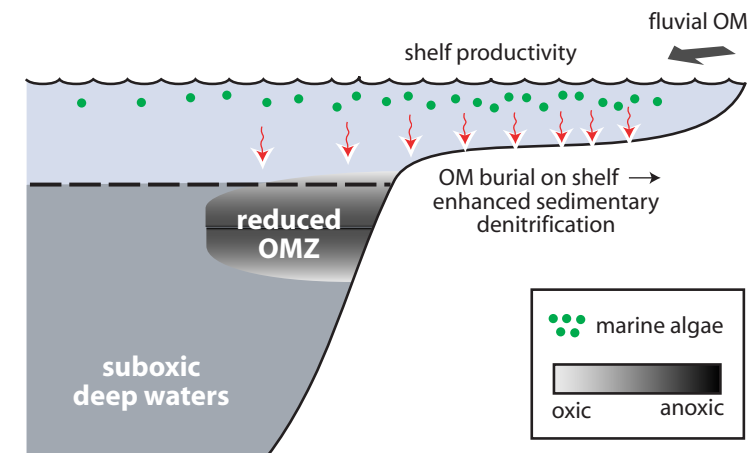

Greenhouse climate mode

b

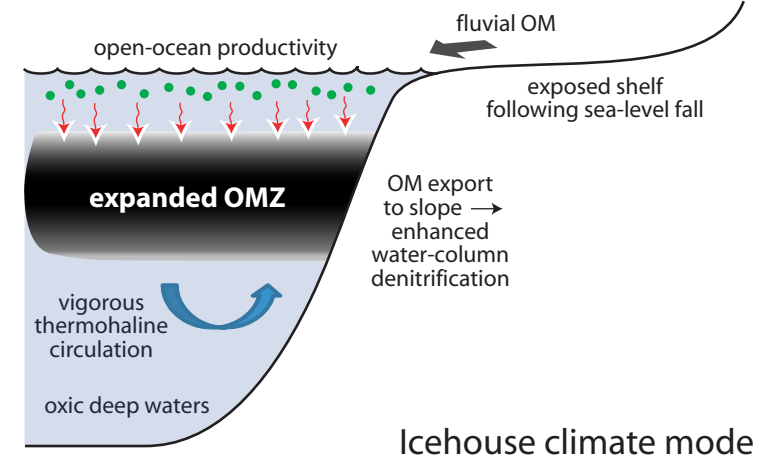

Fig. 9. Model for eustatic control of long-term $\delta^{15} \mathrm{~N}_{\text {sed variation. }}$ (a) Greenhouse climate mode. High eustatic levels result in on-shelf marine productivity and delivery of fluvial organic matter (OM) to shelf environments. Large quantities of OM are trapped in shelf sediments, enhancing sedimentary denitrification. (b) Icehouse climate mode. Low eustatic levels result in open-ocean productivity and delivery of fluvial OM to shelf margins. The sinking flux of OM to the thermocline is elevated, leading to expansion of the oxygenminimum zone (OMZ) and enhanced water-column denitrification.

circulation patterns. The Early Mississippian was a time of closure of an equatorial seaway in the Rheic Ocean region, which probably led to a change from circum-equatorial to meridional ocean circulation (Saltzman, 2003). The Late Cretaceous coincided with widening of the central and south Atlantic basins and a translocation of deepwater formation into the North Atlantic region (MacLeod and Huber, 1996; Barrera et al., 1997; Frank and Arthur, 1999). These examples show that the marine $\mathrm{N}$ cycle is intimately linked to first-order tectonic and climatic cycles, although further investigation will be needed to determine the exact nature of these connections.

The hypothesis that the marine $\mathrm{N}$ cycle has been a driver of long-term climate change is speculative but cannot be dismissed entirely. The critical issue is the nature of links between plate tectonics and global climate. Past work has focused largely on the role of the carbon cycle, i.e., changes

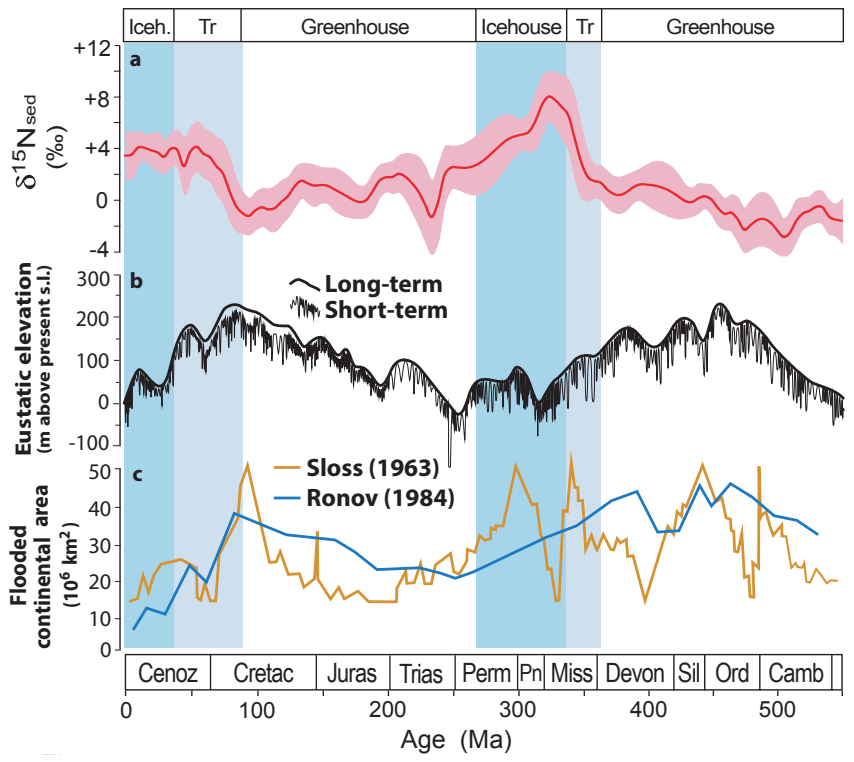

Fig. 10. Comparison of secular variation in $\delta^{15} \mathrm{~N}_{\text {sed }}$ with Phanerozoic eustatic and continental flooding records. (a) $\delta{ }^{15} \mathrm{~N}_{\text {sed }}$ data from Fig. 1. (b) Eustatic data from Haq and Al-Qahtani (2005) and Haq and Schutter (2008) as given in Snedden and Liu (2010). (c) Continental flooding data from Sloss (1963) and Ronov (1984) as given in Miller et al. (2005). Phanerozoic climate modes at top are from Fig. 1; all ages have been adjusted to the Gradstein et al. (2012) timescale. $\operatorname{Tr}=$ transitional interval.

in atmospheric $p \mathrm{CO}_{2}$ linked to mantle degassing, rates of uplift and continental weathering, and changes in marine organic carbon burial rates as a function of oceanic circulation and seawater redox conditions (Mackenzie and Pigott, 1981; Raymo and Ruddiman, 1991; Falkowski et al., 2000; Zachos et al., 2001; Berner, 2006a). The marine $\mathrm{N}$ cycle is intimately connected to burial of marine organic carbon (Gruber, 2004; Galloway et al., 2004), but whether it is a passive responder to changes in carbon fluxes (as generally assumed) or an active control on such changes is uncertain. One mechanism by which the $\mathrm{N}$ cycle might be a driver is through switches between equatorial and polar sites of deepwater formation (Barrera et al., 1997; Frank and Arthur, 1999), with attendant effects on sites of deepwater nutrient upwelling. Even if the marine $\mathrm{N}$ cycle is a passive responder to carbon-cycle forcings, it may play an important role as an amplifier of climate change. For example, enhanced $\mathrm{N}_{2} \mathrm{O}$ production in low-oxygen regions of the ocean during extended intervals of climatic warming might serve as a positive climate feedback (Naqvi et al., 1998; Bakker et al., 2013) that promotes a bimodality of long-term climate conditions (i.e., greenhouse versus icehouse modes; Fig. 1). 


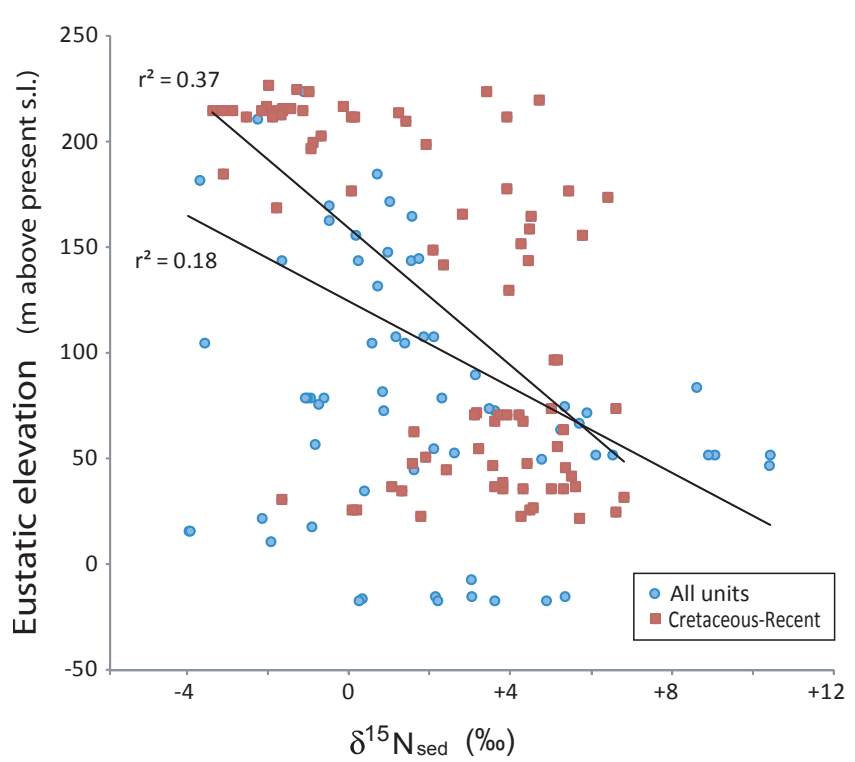

Fig. 11. $\delta^{15} \mathrm{~N}_{\text {sed }}$ versus eustatic elevation (data from Fig. 10). A significant negative relationship exists for all Phanerozoic marine units $\left(r^{2}=0.18 ; n=153 ; p(\alpha)<0.05\right)$, although the relationship is distinctly stronger for Cretaceous-Recent units $\left(r^{2}=0.37, n=\right.$ $84 ; p(\alpha)<0.01)$.

\section{Conclusions}

The present analysis of $\delta^{15} \mathrm{~N}$ variation in 153 marine sedimentary units ranging in age from the Neoproterozoic to the Quaternary is the first to assess long-term variation in the marine $\mathrm{N}$ cycle and controls thereon. Variation in $\delta^{15} \mathrm{~N}_{\text {sed }}$, which serves as a proxy for seawater nitrate $\delta^{15} \mathrm{~N}$, exhibits strong secular coherence since $660 \mathrm{Ma}$, with $74 \%$ of total variance accounted for by a LOWESS trend. This pattern is surprising because the short residence time of fixed $\mathrm{N}$ in modern seawater $(\leq 3 \mathrm{kyr})$ suggests that short-term variation in the marine $\mathrm{N}$ cycle has the potential to dominate the sedimentary $\mathrm{N}$-isotope record and produce no coherent long-term patterns. Average $\delta^{15} \mathrm{~N}_{\text {sed }}$ ranges from lower values $(\sim-2$ to $+2 \%$ ) during greenhouse climate modes of the mid-Paleozoic and mid-Mesozoic to higher $\delta^{15} \mathrm{~N}(\sim+4$ to $+8 \%$ ) during icehouse climate modes of the late Paleozoic and Cenozoic. This pattern suggests that long-term variation in the marine $\mathrm{N}$ cycle is controlled by first-order tectonic cycles, and that it is linked to - and possibly a driver of - longterm climate change. We tentatively link long-term variation in the marine nitrogen cycle to global sea-level changes and shifts in the dominant locus of denitrification, with sedimentary denitrification and water-column denitrification dominant during greenhouse highstands and icehouse lowstands, respectively, a relationship confirmed by reservoir box modeling. These results also challenge the widely held idea that oceanic anoxic events (OAEs) were associated with elevated rates of water-column denitrification. Rather, the present study shows that globally integrated water-column denitrification rates must have been lower during greenhouse intervals (when OAEs developed) relative to icehouse intervals.

\section{Supplementary material related to this article is available online at http://www.biogeosciences.net/11/ 1273/2014/bg-11-1273-2014-supplement.zip.}

Acknowledgements. We thank Craig Johnson, Leo Krystyn, Steven Lev, Jeff Over, Thomas Pedersen, Brad Sageman, Peter Sauer, and Shane Schoepfer for providing nitrogen isotopic data or sample material. We thank Wolfgang Kiessling for editorial handling of the manuscript. T. J. Algeo gratefully acknowledges support from the Sedimentary Geology and Paleobiology program of the US National Science Foundation (grants EAR-0745574 and EAR-1053449), the NASA Exobiology program, and the State Key Laboratory of Geological Processes and Mineral Resources, China University of Geosciences, Wuhan (program GPMR201301).

T. J. Algeo, P. A. Meyers, and R. S. Robinson developed the project, and all authors generated data, participated in data analysis, and drafted the manuscript.

Edited by: W. Kiessling

\section{References}

Algeo, T. J. and Meyers, P. A.: Icehouse-greenhouse climate impacts on the Phanerozoic marine nitrogen cycle, Geol. Soc. Am. Abstr. Progr., 44, p. 458, 2009.

Algeo, T. J., Rowe, H., Hower, J. C., Schwark, L., Hermann, A. and Heckel, P. H.: Oceanic denitrification during Late Carboniferous glacial-interglacial cycles, Nat. Geosci., 1, 709-714, 2008.

Algeo, T. J., Henderson, C. M., Ellwood, B., Rowe, H., Elswick, E., Bates, S., Lyons, T., Hower, J. C., Smith, C., Maynard, J. B., Hays, L. E., Summons, R. E., Fulton, J., and Freeman, K. H.: Evidence for a diachronous Late Permian marine crisis from the Canadian Arctic region, Geol. Soc. Am. Bull., 124, 1424-1448, 2012.

Altabet, M. A.: Variations in nitrogen isotopic composition between sinking and suspended particles: Implications for nitrogen cycling and particle transformation in the open ocean, Deep-Sea Res. I, 35, 535-554, 1988.

Altabet, M. A.: Nitrogen isotopic evidence for micronutrient control of fractional $\mathrm{NO}_{3}^{-}$utilization in the equatorial Pacific, Limnol. Oceanogr., 46, 368-380, 2001.

Altabet, M. A. and François, R.: Sedimentary nitrogen isotopic ratio as a recorder for surface ocean nitrate utilization, Glob. Biogeochem. Cy., 8, 103-116, 1994.

Altabet, M. A., Francois, R., Murray, D. W., and Prell, W. L.: Climate-related variations in denitrification in the Arabian Sea from sediment ${ }^{15} \mathrm{~N} /{ }^{14} \mathrm{~N}$ ratios, Nature, 373, 506-509, 1995.

Bakker, C. C. E., Bange, H. W., Borges, A. V., Delille, B., Gruber, N., Johannessen, T., Löscher, C., Naqvi, S. W. A., Omar, A. M., Santana-Casiano, J. M., and Upstill-Goddard, R. C.: Air-sea interactions of natural long-lived greenhouse gases $\left(\mathrm{CO}_{2}, \mathrm{~N}_{2} \mathrm{O}\right.$, 
$\mathrm{CH}_{4}$ ) in a changing climate, in: Ocean-Atmosphere Interactions of Gases and Particles, edited by: Liss, P. S. and Johnson, M. T., Springer, Berlin, 113-169, 2014.

Barford, C. C., Montoya, J. P., Altabet, M. A., and Mitchell, R.: Steady-state nitrogen isotope effects of $\mathrm{N}_{2}$ and $\mathrm{N}_{2} \mathrm{O}$ production in Paracoccus denitrificans, Appl. Env. Microbiol., 65, 989-994, 1999.

Barrera, E., Savin, S. M., Thomas, E., and Jones, C. E.: Evidence for thermohaline-circulation reversals controlled by sea level change in the latest Cretaceous, Geology, 25, 715-718, 1997.

Beauchamp, B. and Baud, A.: Growth and demise of Permian biogenic chert along northwest Pangea: evidence for end-Permian collapse of thermohaline circulation, Palaeogeogr. Palaeoclimatol. Palaeoecol., 184, 37-63, 2002.

Berner, R. A.: GEOCARBSULF: A combined model for Phanerozoic atmospheric $\mathrm{O}_{2}$ and $\mathrm{CO}_{2}$, Geochim. Cosmochim. Acta, 70, 5653-5664, 2006a.

Berner, R. A: Geological nitrogen cycle and atmospheric $\mathrm{N}_{2}$ over Phanerozoic time, Geology, 34, 413-415, $2006 \mathrm{~b}$.

Brandes, J. A. and Devol, A. H.: Isotopic fractionation of oxygen and nitrogen in coastal marine sediments, Geochim. Cosmochim. Acta, 61, 1793-1801, 1997.

Brandes, J. A. and Devol, A. H.: A global marine-fixed nitrogen isotopic budget: implications for Holocene nitrogen cycling, Glob. Biogeochem. Cy., 16, 1120, doi:10.1029/2001GB001856, 1-14, 2002.

Broecker, W. S. and Henderson, G. M.: The sequence of events surrounding Termination II and their implications for the cause of glacial-interglacial $\mathrm{CO}_{2}$ changes, Paleoceanography, 13, 352364, 1998.

Busigny, V., Cartigny, P., Philippot, P., Ader, M., and Javoy, M.: Massive recycling of nitrogen and other fluid-mobile elements $(\mathrm{K}, \mathrm{Rb}, \mathrm{Cs}, \mathrm{H})$ in a cold slab environment: evidence from HP to UHP oceanic metasediments of the Schistes Lustrés nappe (western Alps, Europe), Earth Planet. Sci. Lett., 215, 27-42, 2003.

Carpenter, E. J., Harvey, H. R., Fry, B., and Capone, D. G.: Biogeochemical tracers of the marine cyanobacterium Trichodesmium, Deep-Sea Res. I, 44, 27-38, 1997.

Cleveland, W. S., Grosse, E., and Shyu, W. M.: Local regression models, in: Statistical Models in S, edited by: Chambers, J. M. and Hastie, T., Chapman Hall, Boca Raton, Florida, 309-376, 1992.

Codispoti, L.: Is the ocean losing nitrate?, Nature, 376, 724, 1995.

Codispoti, L. A.: An oceanic fixed nitrogen sink exceeding $400 \mathrm{Tg}$ $\mathrm{N}^{a-1}$ vs the concept of homeostasis in the fixed-nitrogen inventory, Biogeosciences, 4, 233-253, doi:10.5194/bg-4-233-2007, 2007.

Deutsch, C., Sigman, D. M., Thunell, R. C., Meckler, N., and Haug, G. H.: Isotopic constraints on the glacial/interglacial oceanic nitrogen budget, Glob. Biogeochem. Cy., 18, 1-22, 2004.

Deutsch, C., Sarmiento, J. L., Sigman, D. M., Gruber, N., and Dunne, J. P.: Spatial coupling of nitrogen inputs and losses in the ocean, Nature, 445, 163-167, 2007.

DeVries, T., Deutsch, C., Primeau, F., Chang, B., and Devol, A.: Global rates of water-column denitrification derived from nitrogen gas measurements, Nat. Geosci., 5, 547-550, 2012.

DeVries, T., Deutsch, C., Rafter, P. A., and Primeau, F.: Marine denitrification rates determined from a global 3-D inverse model,
Biogeosciences, 10, 2481-2496, doi:10.5194/bg-10-2481-2013, 2013.

Ertel, J. R. and Hedges, J. I.: Sources of sedimentary humic substances: vascular plant debris, Geochim. Cosmochim. Acta, 49, 2097-2107, 1985.

Espitalié, J., Laporte, J. L., Madec, M., Marquis, F., Leplat, P., Paulet, J., and Boutefeu, A.: Méthode rapide de caractérisation des roches mères de leur potentiel pétrolier et de leur degré d'évolution, Rev. Instit. Franç. Pétrole, 32, 23-42, 1977.

Espitalié, J., Deroo, G., and Marquis, F.: Rock Eval pyrolysis and its applications, Rev. Instit. Franç. Pétrole, Part I: 32, 653-578; Part II: 40, 755-784; Part III: 41, 73-89, 1985.

Eugster, O. and Gruber, N.: A probabilistic estimate of global marine $\mathrm{N}$-fixation and denitrification, Glob. Biogeochem. Cy., 26, GC4013, doi:10.1029/2012GB004300, 2012.

Falkowski, P., Scholes, R. J., Boyle, E., Canadell, J., Canfield, D., Elser, J., Gruber, N., Hibbard, K., Högberg, P., Linder, S., Mackenzie, F. G., Moore, B., III, Pedersen, T., Rosenthal, Y., Seitzinger, S. Smetacek, V., and Steffen, W.: The global carbon cycle: A test of our knowledge of Earth as a system, Science, 290, 291-296, 2000.

François, R., Altabet, M. A., and Burckle, L. H.: Glacial to interglacial changes in surface nitrate utilization in the Indian section of the Southern Ocean as recorded by sediment $\delta^{15} \mathrm{~N}$, Paleoceanography, 7, 589-606, 1992.

Frank, T. D. and Arthur, M. A.: Tectonic forcings of Maastrichtian ocean-climate evolution. Paleoceanography, 14, 103-117, 1999.

Frimmel, A., Oschmann, W., and Schwark, L.: Chemostratigraphy of the Posidonia Black Shale, SW-Germany: I - Influence of sea level variation on organic facies evolution, Chem. Geol., 206, 199-230, 2004.

Galbraith, E. D., Kienast, M., Pedersen, T. F., and Calvert, S. E.: Glacial-interglacial modulation of the marine nitrogen cycle by high-latitude $\mathrm{O}_{2}$ supply to the global thermocline, Paleoceanography, 19, 1-12, 2004.

Galbraith, E. D., Sigman, D. M., Robinson, R. S., and Pedersen, T. F.: Nitrogen in past marine environments, in: Nitrogen in the Marine Environment, 2nd ed., edited by: Capone, D. G., Bronk, D. A., Mulholland, M. R., and Carpenter, E. J., Elsevier, Amsterdam, 1497-1535, 2008.

Galloway, J. N., Dentener, F. J., Capone, D. G., Boyer, E. W., Howarth, R. W., Seitzinger, S. P., Asner, G. P., Cleveland, C. C., Green, P. A., Holland, E. A., Karl, D. M., Michaels, A. F., Porter, J. H., Townsend, A. R., and Vörösmarty, C. J.: Nitrogen cycles: past, present, and future, Biogeochemistry, 70, 153-226, 2004.

Ganeshram, R. S., Pedersen, T. F., Calvert, S. E., and Murray, J. W.: Large changes in oceanic nutrient inventories from glacial to interglacial periods, Nature, 376, 755-758, 1995.

Gaye-Haake, B., Lahajnar, N., Emeis, K.-C., Unger, D., Rixen, T., Suthhof, A., Ramaswamy, V., Schulz, H., Paropkari, A. L., Guptha, M. V. S. and Ittekkot, V.: Stable nitrogen isotopic ratios of sinking particles and sediments from the northern Indian Ocean, Mar. Chem., 96, 243-255, 2005.

Gradstein, F. M., Ogg, J. G., Schmitz, M. D., and Ogg, G. M. (Eds.): The Geologic Time Scale 2012, 2 vols., Elsevier, Amsterdam, 1144 pp., 2012.

Granger, J., Sigman, D. M., Rohde, M. M., Maldonado, M. T., and Tortell, P. D.: N and $\mathrm{O}$ isotope effects during nitrate assimila- 
tion by unicellular prokaryotic and eukaryotic plankton cultures, Geochim. Cosmochim. Acta, 74, 1030-1040, 2010.

Grice, K., Cao, C., Love, G. D., Böttcher, M. E., Twitchett, R. J., Grosjean, E., Summons, R. E., Turgeon, S. C., Dunning, W., and Jin, Y.: Photic zone euxinia during the Permian-Triassic superanoxic event, Science, 307, 706-709, 2005.

Grosskopf, T., Mohr, W., Baustian, T., Schunck, H., Gill, D., Kuypers, M. M. M., Lavik, G., Schmitz, R. A., Wallace, D. W. R., and LaRoche, J.: Doubling of marine dinitrogen-fixation rates based on direct measurements, Nature, 488, 361-364, 2012.

Gruber, N. G.: The dynamics of the marine nitrogen cycle and its influence on atmospheric $\mathrm{CO}_{2}$ variations, in: The Ocean Carbon Cycle and Climate, edietd by: Follows, M. and Oguz, T., Kluwer, Dordrecht, 97-148, 2004.

Gruber, N. G.: The marine nitrogen cycle: overview and challenges, in: Nitrogen in the Marine Environment, 2nd Edn., edited by: Capone, D. G., Bronk, D. A., Mulholland, M. R., and Carpenter, E. J., Elsevier, Amsterdam, 1-50, 2008.

Gruber, N. G. and Galloway, J. N.: An Earth-system perspective of the global nitrogen cycle, Nature, 451, 293-296, 2008.

Gruber, N. and Sarmiento, J.: Global patterns of marine nitrogen fixation and denitrification, Glob. Biogeochem. Cy., 11, 235-266, 1997.

Haq, B. U. and Al-Qahtani, A. M.: Phanerozoic cycles of sea-level change on the Arabian Platform, GeoArabia, 10, 127-160, 2005.

Haq, B. U. and Schutter, S. R.: A chronology of Paleozoic sea-level changes, Science, 322, 64-68, 2008.

Hartnett, H. E., Keil, R. G., Hedges, J. I., and Devol, A. H.: Influence of oxygen exposure time on organic carbon preservation in continental margin sediments, Nature, 391, 572-574, 1998.

Haug, G. H., Pedersen, T. F., Sigman, D. M., Calvert, S. E., Nielsen, B., and Peterson, L. C.: Glacial/interglacial variations in production and nitrogen fixation in the Cariaco Basin during the last 580 kyr, Paleoceanography, 13, 427-432, 1998.

Hedges, J. I., Keil, R. G., and Benner, R.: What happens to terrestrial organic matter in the ocean?, Org. Geochem., 27, 195-212, 1997.

Higgins, M. B., Robinson, R. S., Carter, S. J., and Pearson, A.: Evidence from chlorin nitrogen isotopes for alternating nutrient regimes in the Eastern Mediterranean Sea, Earth Planet. Sci. Lett., 290, 102-107, 2010.

Higgins, M. D., Robinson, R. S., Husson, J. M., Carter, S. J., and Pearson, A.: Dominant eukaryotic export production during ocean anoxic events reflects the importance of recycled $\mathrm{NH}_{4}^{+}$, Proc. Nat. Acad. Sci. (USA), 109, 2269-2274, 2012.

Hoch, M. P., Fogel, M. L., and Kirchman, D. L.: Isotope fractionation during ammonium uptake by marine microbial assemblages, Geomicrobiol. J., 12, 113-127, 1994.

Holloway, J. M. and Dahlgren, R. A.: Geologic nitrogen in terrestrial biogeochemical cycling, Geology, 27, 567-570, 1999.

Holloway, J. M., Dahlgren, R. A., Hansen, B., and Casey, W. H.: Contribution of bedrock nitrogen to high nitrate concentrations in stream water, Nature, 395, 785-788, 1998.

Holmes, M. E., Eichner, C., Struck, U., and Wefer, G.: Reconstruction of surface ocean nitrate utilization using stable nitrogen isotopes in sinking particles and sediments, in: Fischer, G. and Wefer, G. (Eds.), Use of Proxies in Paleoceanography: Examples from the South Atlantic, Springer, Berlin, 447-468, 1999.
Huang, W. Y. and Meinschein, W. G.: Sterols as ecological indicators, Geochim. Cocmochim. Acta, 43, 739-745, 1979.

Hutton, A. C.: Petrographic classification of oil shales, Internat. J. Coal Geol., 8, 203-231, 1987.

Imbus, S. W., Macko, S. A., Elmore, R. D., and Engel, M. H.: Stable isotope (C, S, N) and molecular studies on the Precambrian Nonesuch Shale (Wisconsin-Michigan, USA): evidence for differential preservation rates, depositional environment and hydrothermal influence, Chem. Geol., 101, 255-281, 1992.

Jaminski, J., Algeo, T. J., Maynard, J. B., and Hower, J. C.: Climatic origin of dm-scale compositional cyclicity in the Cleveland Member of the Ohio Shale (Upper Devonian), Central Appalachian Basin, USA, in: Shales and Mudstones, v. 1, Schieber, J., Zimmerle, W., and Sethi, P. S., Schweizerbart'sche, Stuttgart, 217-242, 1998.

Jenkyns, H. C., Gröcke, D. R., and Hesselbo, S. P.: Nitrogen isotope evidence for water mass denitrification during the early Toarcian (Jurassic) oceanic anoxic event, Paleoceanography, 16, 593-603, 2001.

Jenkyns, H. C., Matthews, A., Tsikos, H., and Erel, Y.: Nitrate reduction, sulfate reduction, and sedimentary iron isotope evolution during the Cenomanian-Turonian oceanic anoxic event, $\mathrm{Pa}-$ leoceanography, 22, 1-17, 2007.

Jia, Y. and Kerrich, R.: Nitrogen 15-enriched Precambrian kerogen and hydrothermal systems, Geochem. Geophys. Geosyst., 5, 121, 2004.

Junium, C. K. and Arthur, M. A.: Nitrogen cycling during the Cretaceous, Cenomanian-Turonian Oceanic Anoxic Event II, Geochem. Geophys. Geosyst., 8, 1-18, 2007.

Kenrick, P. and Crane, P. R.: The origin and early evolution of plants on land, Nature, 389, 33-39, 1997.

Knapp, A. N., DiFiore, P. J., Deutsch, C., Sigman, D. M., and Lipschultz, F.: Nitrate isotopic composition between Bermuda and Puerto Rico: implications for $\mathrm{N}_{2}$ fixation in the Atlantic Ocean, Glob. Biogeochem. Cy., 22, 1-14, 2008.

Kritee, K., Sigman, D. M., Granger, J., Ward, B. B., Jayakumar, A., and Deutsch, C.: Reduced isotope fractionation by denitrification under conditions relevant to the ocean, Geochim. Cosmochim. Acta, 92, 243-259, 2012.

Kuypers, M. M. M., Lavik, G., Woebken, D., Schmid, M., Fuchs, B. M., Amann, R., Jorgensen, B. B., and Jetten, M. S. M.: Massive nitrogen loss from the Benguela upwelling system through anaerobic ammonium oxidation, Proc. Natl. Acad. Sci. (USA), 102, 6478-6483, 2005.

Lehmann, M. F., Sigman, D. M., and Berelson, W. M.: Coupling the $\mathrm{N}-15 / \mathrm{N}-14$ and $\mathrm{O}-18 / \mathrm{O}-16$ of nitrate as a constraint on benthic nitrogen cycling, Mar. Chem., 88, 1-20, 2004.

Lehmann, M. F., Sigman, D. M., McCorkle, D. C., Granger, J., Hoffmann, S., Cane, G., and Brunelle, B. G.: The distribution of nitrate ${ }^{15} \mathrm{~N} /{ }^{14} \mathrm{~N}$ in marine sediments and the impact of benthic nitrogen loss on the isotopic composition of oceanic nitrate, Geochim. Cosmochim. Acta, 71, 5384-5404, 2007.

Libes, S. M. and Deuser, W. G.: The isotopic geochemistry of particulate nitrogen in the Peru Upwelling Area and the Gulf of Maine, Deep-Sea Res. I, 35, 517-533, 1988.

Liu, Z., Altabet, M. A., and Herbert, T. D.: Glacial-interglacial modulation of eastern tropical North Pacific denitrification over the last 1.8-Myr, Geophys. Res. Lett., 32, 1-4, 2005. 
Liu, Z., Altabet, M. A., and Herbert, T. D.: Plio-Pleistocene denitrification in the eastern tropical North Pacific: intensification at 2.1 Ma, Geochem. Geophys. Geosyst., 9, 1-14, 2008.

Lourey, M. J., Trull, T. W. and Sigman, D. M.: Sensitivity of $\delta^{15} \mathrm{~N}$ of nitrate, surface suspended and deep sinking particulate nitrogen to seasonal nitrate depletion in the Southern Ocean, Glob. Biogeochem. Cy., 17, 1-18, 2003.

Lücke, A. and Brauer, A.: Biogeochemical and micro-facial fingerprints of ecosystem response to rapid Late Glacial climatic changes in varved sediments of Meerfelder Maar (Germany), Palaeogeogr. Palaeoclimatal. Palaeoecol., 211, 139-155, 2004.

Luo, G., Wang, Y., Kump, L. R., Bai, X., Algeo, T. J., Yang, H., and Xie, S.: Enhanced nitrogen fixation in the aftermath of the latest Permian marine mass extinction, Geology, 39, 647-650, 2011.

Mackenzie, F. T. and Pigott, J. D.: Tectonic controls of Phanerozoic sedimentary rock cycling, J. Geol. Soc. London, 138, 183-196, 1981.

Macko, S. A. and Estep, M. L. F.: Microbial alteration of stable nitrogen and carbon isotopic compositions of organic matter, Org. Geochem., 6, 787-790, 1984.

Macko, S. A., Estep, M. L. F., Engel, M. H., and Hare, P. E.: Kinetic fractionation of stable nitrogen isotopes during amino acid transamination, Geochim. Cosmochim. Acta, 50, 2143-2146, 1986.

Macko, S. A., Fogel, M. L., Hare, P. E., and Hoering, T. C.: Isotopic fractionation of nitrogen and carbon in the synthesis of amino acids by microorganisms, Chem. Geol., 65, 79-92, 1987.

MacLeod, K. G. and Huber, B. T.: Reorganization of deep ocean circulation accompanying a Late Cretaceous extinction event, Nature, 380, 422-425, 1996. Mariotti, A.: Natural ${ }^{15} \mathrm{~N}$ abundance measurements and atmospheric nitrogen standard calibration, Nature, 311, 251-252, 1984.

Meyers, P. A.: Preservation of elemental and isotopic source identification of sedimentary organic matter, Chem. Geol., 114, 289302, 1994.

Meyers, P. A.: Organic geochemical proxies of paleoceanographic, paleolimnologic, and paleoclimatic processes, Org. Geochem., 27, 213-250, 1997.

Meyers, P. A., Yum, J.-G., and Wise, S. W.: Origins and maturity of organic matter in mid-Cretaceous black shales from ODP Site 1138 on the Kerguelen Plateau, Mar. Petrol. Geol., 26, 909-915, 2009a.

Meyers, P. A., Bernasconi, S. M., and Yum, J.-G.: 20 My of nitrogen fixation during deposition of mid-Cretaceous black shales on the Demerara Rise, equatorial Atlantic Ocean, Org. Geochem., 40, 158-166, 2009b.

Middelburg, J. J., Soetaert, K., Herman, P. M. J., and Heip, C. H. R.: Denitrification in marine sediments: a model study, Glob. Biogeochem. Cy., 10, 661-673, 1996.

Mii, H.-S., Grossman, E. L., Yancey, T. E., Chuvashov, B., and Egorov, A.: Isotopic records of brachiopod shells from the Russian Platform - evidence for the onset of mid-Carboniferous glaciation, Chem. Geol., 175, 133-147, 2001.

Miller, K. G., Kominz, M. A., Browning, J. V., Wright, J. D., Mountain, G. S., Katz, M. E., Sugarman, P. J., Cramer, B. S., ChristieBlick, N., and Pekar, S. F.: The Phanerozoic record of global sea-level change, Science, 310, 1293-1298, 2005.

Montañez, I. P. Norris, R. D., Algeo, T. J., Chandler, M. A., Johnson, K. R., Kennedy, M. J., Kent, D. V., Kiehl, J. T., Kump, L.
R., Ravelo, A. C., and Turekian, K. K.: Understanding Earth's Deep Past: Lessons for Our Climate Future, National Academy of Sciences, Washington, DC, 212 pp., 2011.

Mulder, A., van de Graaf, A. A., Robertson, L. A., and Kuenen, J. G.: Anaerobic ammonium oxidation discovered in a denitrifying fluidized bed reactor, FEMS Microbiol. Ecol., 16, 177-184, 1995.

Müller, P. J.: C/N ratios in Pacific deep-sea sediments: Effect of inorganic ammonium and organic nitrogen compounds sorbed by clays, Geochim. Cosmochim. Acta, 41, 765-776, 1977.

Murray, J. W., Fuchsman, C., Kirkpatrick, J., Paul, B., and Konovalov, S. K.: Species and $\delta^{15} \mathrm{~N}$ signatures of nitrogen transformations in the suboxic zone of the Black Sea, Oceanography, 18, 36-47, 2005.

Naqvi, S. W. A., Yoshinari, T., Jayakumar, D. A., Altabet, M. A., Narvekar, P. V., Devol, A. H., Brandes, J. A., and Codispoti, L. A.: Budgetary and biogeochemical implications of $\mathrm{N}_{2} \mathrm{O}$ isotope signatures in the Arabian Sea, Nature, 394, 462-464, 1998.

Ogrinc, N., Fontolan, G., Faganeli, J., and Covelli, S.: Carbon and nitrogen isotope compositions of organic matter in coastal marine sediments (the Gulf of Trieste, $\mathrm{N}$ Adriatic Sea): indicators of sources and preservation, Mar. Chem., 95, 163-181, 2005.

Peters, K. E.: Guidelines for evaluating petroleum source rock using programmed pyrolysis, Am. Assoc. Petrol. Geol. Bull., 70, 318329, 1986.

Peters, K. E., Walters, C. C., and Moldowan, J. M., The Biomarker Guide, 2nd Edn., 2 vols., Cambridge University Press, Cambridge, 2004.

Prahl, F. G., de Lange, G. J., Scholten, S., and Cowie, G. L.: A case of post-depositional aerobic degradation of terrestrial organic matter in turbidite deposits from the Madeira abyssal plain, Org. Geochem., 27, 141-152, 1997.

Prokopenko, M. G., Hammond, D. E., Berelson, W. M., Bernhard, J. M., Stott, L., and Douglas, R.: Nitrogen cycling in the sediments of Santa Barbara basin and Eastern Subtropical North Pacific: nitrogen isotopes, diagenesis and possible chemosymbiosis between two lithotrophs (Thioploca and Anammox) - "riding on a glider”, Earth Planet. Sci. Lett., 242, 186-204, 2006.

Rau, G. H., Arthur, M. A., and Dean, W. E.: ${ }^{15} \mathrm{~N} /{ }^{14} \mathrm{~N}$ variations in Cretaceous Atlantic sedimentary sequences: Implication for past changes in marine nitrogen biogeochemistry, Earth Planet. Sci. Lett., 82, 269-279, 1987.

Raymo, M. E. and Ruddiman, W. F.: Tectonic forcing of late Cenozoic climate, Nature, 359, 117-122, 1991.

Robinson, R. S. and Meyers, P. A.: Biogeochemical changes within the Benguela Current upwelling system during the Matuyama Diatom Maximum: Nitrogen isotope evidence from Ocean Drilling Program Sites 1082 and 1084, Paleoceanography, 17, 1-10, 2002.

Robinson, R. S., Sigman, D. M., DiFiore, P. J., Rohde, M. M., Mashiotta, T. A., and Lea, D. W.: Diatom-bound ${ }^{15} \mathrm{~N} /{ }^{14} \mathrm{~N}$ : New support for enhanced nutrient consumption in the ice age subantarctic, Paleoceanography, 20, 1-14, 2005.

Robinson, R. S. and 30 coauthors: A review of nitrogen isotope alteration in marine sediments, Paleoceanography, 27, 1-13, 2012.

Romano, C., Goudemand, N., Vennemann, T. W., Ware, D., Schneebeli-Hermann, E., Hochuli, P. A., Brühwiler, T., Brinkmann, W., and Bucher, H.: Climatic and biotic upheavals 
following the end-Permian mass extinction, Nat. Geosci., 6, 5760, 2012.

Ronov, A. B.: Phanerozoic transgressions and regressions on the continents: A quantitative approach based on areas flooded by the sea and areas of marine and continental deposition, Am. J. Sci., 294, 777-801, 1984.

Sachs, J. P. and Repeta, D. J.: Oligotrophy and nitrogen fixation during Eastern Mediterranean sapropel events, Science, 286, 24852488, 1999.

Sachs, J. P., Repeta, D. J., and Goericke, R.: Nitrogen and carbon isotopic ratios of chlorophyll from marine phytoplankton, Geochim. Cosmochim. Acta, 63, 1431-1441, 1999.

Saino, T.: ${ }^{15} \mathrm{~N}$ and ${ }^{13} \mathrm{C}$ natural abundance in suspended particulate organic matter from a Kuroshio warm-core ring, Deep-Sea Res. I, 39, 347-362, 1992.

Saltzman, M. R.: Late Paleozoic ice age: oceanic gateway or $p \mathrm{CO}_{2}$ ?, Geology, 31, 151-154, 2003.

Schoepfer, S. D., Henderson, C. M., Garrison, G. H., and Ward, P. D.: Cessation of a productive coastal upwelling system in the Panthalassic Ocean at the Permian-Triassic boundary, Palaeogeogr. Palaeoclimatol. Palaeoecol., 313/314, 181-188, 2012.

Schoepfer, S. D., Henderson, C. M., Garrison, G. H., Ward, P. D., Foriel, J., Selby, D., Hower, J. C., Algeo, T. J., and Shen, Y.: Termination of a continent-margin upwelling system at the PermianTriassic boundary (Opal Creek, Alberta, Canada), Glob. Planet. Change, 105, 21-35, 2013.

Sephton, M. A., Looy, C. V., Brinkhuis, H., Wignall, P. B., de Leeuw, J. W., and Visscher, H.: Catastrophic soil erosion during the end-Permian biotic crisis, Geology, 33, 941-944, 2005.

Sigman, D. M., Altabet, M. A., François, R., McCorkle, D. C., and Gaillard, J.-F.: The isotopic composition of diatom-bound nitrogen in Southern Ocean sediments, Paleoceanography, 14, 118134, 1999

Sigman, D. M., Altabet, M. A., McCorkle, D. C., François, R., and Fischer, G.: The $\delta^{15} \mathrm{~N}$ of nitrate in the Southern Ocean: nitrogen cycling and circulation in the ocean interior, J. Geophys. Res., 105, 19599-19614, 2000

Sigman, D. M., Robinson, R., Knapp, A. N., van Geen, A., McCorkle, D. C., Brandes, J. A., and Thunell, R. C.: Distinguishing between water column and sedimentary denitrification in the Santa Barbara Basin using the stable isotopes of nitrate, Geochem. Geophys. Geosyst., 4, 1-20, 2003.

Sloss, L. L.: Sequences in the cratonic interior of North America. Geol. Soc. Am. Bull., 74, 93-114, 1963.

Snedden, J. W. and Liu, C. J.: A compilation of Phanerozoic sealevel change, coastal onlaps and recommended sequence designations, Am. Assoc. Petrol. Geol. Search and Discovery Article \#40594, 3 pp., 2010.
Somes, C. J., Schmittner, A., and Altabet, M. A.: Nitrogen isotope simulations show the importance of atmospheric iron deposition for nitrogen fixation across the Pacific Ocean, Geophys. Res. Lett., 37, 1-6, 2010.

Song, H. Y., Tong, J. N., Algeo, T. J., Horacek, M., Qiu, H. O., Song, H. J., Tian, L., and Chen, Z. Q.: Large vertical $\delta^{13} \mathrm{C}$ gradients in Early Triassic seas of the South China craton: Implications for oceanographic changes related to Siberian Traps volcanism, Glob. Planet. Change, 105, 7-20, 2013.

Sun, Y., Joachimski, M. M., Wignall, P. B., Yan, C., Chen, Y., Jiang, H., Wang, L., and Lai, X.: Lethally hot temperatures during the Early Triassic greenhouse, Science, 338, 366-370, 2012.

Suthhof, A., Ittekkot, V., and Gaye-Haake, B.: Millennial-scale oscillation of denitrification intensity in the Arabian Sea during the late Quaternary and its potential influence on atmospheric $\mathrm{N}_{2} \mathrm{O}$ and global climate, Glob. Biogeochem. Cy., 15, 637-649, 2001.

Tesdal, J. E., Galbraith, E. D., and Kienast, M.: The marine sedimentary nitrogen isotope record, Biogeosci. Disc., 9, 4067-4097, 2012.

Thamdrup, B., Dalsgaard, T., Jensen, M. M., Ulloa, O., Farías, L., and Escribano, R.: Anaerobic ammonium oxidation in the oxygen-deficient waters off northern Chile, Limnol. Oceanogr., 51, 2145-2156, 2006

Thornton, S. F. and McManus, J.: Application of organic carbon and nitrogen stable isotopes and $\mathrm{OC} / \mathrm{TN}$ ratios as source indicators of OM provenance in estuarine systems: evidence from the Tay Estuary, Scotland, Estuar. Coast. Shelf Sci., 38, 219-233, 1994.

Tyrrell, T.: The relative influences of nitrogen and phosphorus on oceanic primary productivity, Nature, 400, 525-531, 1999.

Voss, M., Dippner, J. W., and Montoya, J. P.: Nitrogen isotope patterns in the oxygen-deficient waters of the Eastern Tropical North Pacific Ocean, Deep-Sea Res. I, 48, 1905-1921, 2001.

Wang, C. J. and Visscher, H.: Abundance anomalies of aromatic biomarkers in the Permian-Triassic boundary section at Meishan, China-Evidence of end-Permian terrestrial ecosystem collapse, Palaeogeogr. Palaeoclimatol. Palaeoecol., 252, 291-303, 2007.

Waser, N. A. D., Harrison, P. J., Nielsen, B., Calvert, S. E., and Turpin, D. H.: Nitrogen isotope fractionation during the uptake and assimilation of nitrate, nitrite, ammonium and urea by a marine diatom, Limnol. Oceanogr., 43, 215-224, 1998.

Xie, S., Pancost, R. D., Huang, J., Wignall, P. B., Yu, J., Tang, X., Chen, L., Huang, X., and Lai, X.: Changes in the global carbon cycle occurred as two episodes during the Permian-Triassic crisis, Geology, 35, 1083-1086, 2007.

Zachos, J., Pagani, M., Sloan, L., Thomas, E., and Billups, K.: Trends, rhythms, and aberrations in global climate $65 \mathrm{Ma}$ to present, Science, 292, 686-693, 2001. 


\section{Appendix A}

\section{Stable isotopic analyses}

For most analyses original to this study, $\mathrm{C}$ and $\mathrm{N}$ elemental and stable isotopic compositions were determined at the University of Texas at Arlington Stable Isotope Laboratory using a Costech 5010 elemental analyzer coupled via a ConfloIII device to a ThermoFinnigan Delta PlusXP isotope-ratio mass spectrometer. Analytical precision was $0.08 \%$ for $\% \mathrm{~N}$, $0.07 \%$ for $\% \mathrm{C}, 0.05 \%$ o for $\delta^{15} \mathrm{~N}$, and $0.09 \%$ or $\delta^{13} \mathrm{C}$ of the laboratory standards. All isotopic results are reported relative to air $\left(\delta^{15} \mathrm{~N}\right)$ and V-PDB $\left(\delta^{13} \mathrm{C}\right)$. A subset of samples used in this study was analyzed in the stable isotope laboratory at the University of Arizona.

\section{Appendix B}

\section{Calculation of LOWESS trend}

Long-term secular variation in our $\delta^{15} \mathrm{~N}_{\text {sed }}$ data set was modeled using a LOWESS (LOcally WEighted Scatterplot Smoothing) procedure, which determines a best-fit trend for irregularly distributed time series data using an inversedistance-squared weight function (Cleveland et al., 1992). The LOWESS curve of Fig. 1a was calculated as

$$
\begin{gathered}
\delta^{15} \mathrm{~N}_{t}=\Sigma\left(1 /\left(1+\left(\left(t-t_{i}\right) / \lambda\right)^{2}\right) \times \delta^{15} \mathrm{~N}_{i}\right) / \\
\Sigma\left(1 /\left(1+\left(\left(t-t_{i}\right) / \lambda\right)^{2}\right)\right)
\end{gathered}
$$

where $\delta^{15} \mathrm{~N}_{t}$ is the estimated mean $\delta^{15} \mathrm{~N}$ at a given time $t, \delta^{15} \mathrm{~N}_{i}$ and $t_{i}$ are the $\mathrm{N}$-isotopic composition and age of study unit $i$, and the summation was performed for $i=1$ to 153 (i.e., the full $\delta^{15} \mathrm{~N}_{\text {sed }}$ data set). The parameter $\lambda$ determines the temporal range (or window) of the inversedistance-squared weight function. Larger values of $\lambda$ are appropriate for intervals of lower data density and will produce a smoother LOWESS curve. We varied $\lambda$ from $10 \mathrm{Myr}$ at 660 to $2 \mathrm{Myr}$ at $0 \mathrm{Ma}$ to match temporal variation in data density. The standard deviation range for $\delta^{15} \mathrm{~N}$ at a given time $t$ was calculated as the mean plus or minus the standard deviation $(\sigma)$, with the latter determined as

$$
\begin{aligned}
& \sigma\left(\delta^{15} N_{t}\right)=\left(\Sigma \left(\left(\delta^{15} N_{t}-\delta^{15} N_{i}\right)^{2} \times\left(1 /\left(1+\left(\left(t-t_{i}\right) / \lambda\right)^{2}\right)\right)\right.\right. \\
& \left.\times \xi) / \Sigma\left(1 /\left(1+\left(\left(t-t_{i}\right) / \lambda\right)^{2}\right)\right)\right)^{0.5}
\end{aligned}
$$

where $\xi$ is the fractional standard deviation range of interest (i.e., 0.68 for $\pm 1 \sigma, 0.95$ for $\pm 2 \sigma$ ). This range is shown as the shaded field bracketing the LOWESS curve in Fig. 1a.
Table C1. Flux constants $\left(k_{i}\right)$ for reservoir model fluxes.

\begin{tabular}{ll}
\hline FLUX & $k_{i}$ \\
\hline$f_{\mathrm{VOL}}$ & $5.63 \mathrm{E}-04$ \\
$f_{\mathrm{RIV}}$ & $3.13 \mathrm{E}-04$ \\
$f_{\mathrm{DT}}$ & $6.25 \mathrm{E}-04$ \\
$f_{\mathrm{DW}} *$ & $7.48 \mathrm{E}-05$ \\
$f_{\mathrm{DS}}$ & $2.00 \mathrm{E}-04$ \\
$f_{\mathrm{W}}$ & $3.57 \mathrm{E}-08$ \\
$f_{\mathrm{FIX}-\mathrm{T}}$ & $3.08 \mathrm{E}-08$ \\
$f_{\mathrm{DEP}}$ & $6.41 \mathrm{E}-09$ \\
$f_{\mathrm{FIX}}$ & $5.00 \mathrm{E}-08$ \\
$f_{\mathrm{BUR}}$ & $3.13 \mathrm{E}-05$ \\
\hline All values as fraction of \\
reservoir mass; ${ }^{*}$ includes \\
$f_{\mathrm{ANX}}$
\end{tabular}

\section{Appendix C}

\section{Marine nitrogen cycle modeling}

The $\mathrm{N}$-isotopic composition of seawater nitrate $\left(\delta^{15} \mathrm{~N}_{\mathrm{NO}_{3}^{-}}\right)$ can be approximated from a steady-state isotope mass balance that assumes $\mathrm{N}$ fixation $\left(f_{\mathrm{FIX}}\right)$ as the primary source and sedimentary $\left(f_{\mathrm{DS}}\right)$ and water-column $\left(f_{\mathrm{DW}}\right)$ denitrification as the two largest sinks for seawater fixed $\mathrm{N}$ (Brandes and Devol, 2002; Deutsch et al., 2004; Gruber, 2008). We developed two reservoir box models of the marine $\mathrm{N}$ cycle: (1) a fully integrated version that incorporates all significant $\mathrm{N}$ reservoirs and fluxes in the Earth's exogenic system (Fig. C1a), and (2) a simplified version that includes only the most important source (i.e., cyanobacterial $\mathrm{N}$ fixation) and sink fluxes (sedimentary and water-column denitrification) for seawater nitrate (Fig. C1b). The two models yield similar results for $\delta^{15} \mathrm{~N}_{\mathrm{NO}_{3}^{-}}$under a wide range of conditions (see below), indicating that the simplified model provides a reasonably robust representation of the major influences on the $\mathrm{N}$-isotopic composition of seawater nitrate. Because the fully integrated model will be developed further in a later study, our focus here is on the results of the simplified model.

In both models, all fluxes are first-order functions of source reservoir mass:

$f_{i}=k_{i} \times M_{x}(t)$,

where $f_{i}$ is the flux of interest, $k_{i}$ its flux constant (Table $\mathrm{C} 1$ ), and $M_{x}$ the mass of source reservoir $x$ at a given time $t$. Temporal changes in reservoir mass from $t$ to $t+1$ 
a

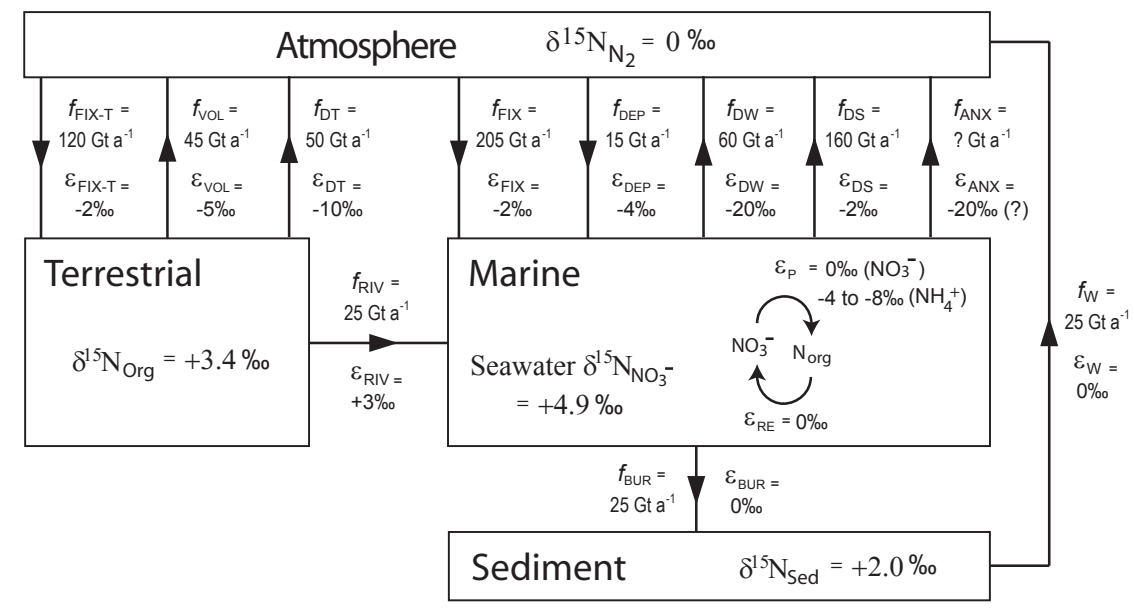

\begin{tabular}{|c|c|c|}
\hline Atmosphere & \multicolumn{2}{|c|}{$\delta^{15} \mathrm{~N}_{\mathrm{N}_{2}}=0 \%$} \\
\hline $\begin{array}{c}f_{\mathrm{FIX}}= \\
205 \mathrm{Gta}^{-1}\end{array}$ & $\begin{array}{l}f_{\mathrm{DW}}= \\
60 \mathrm{Gta}^{-1}\end{array}$ & $\begin{array}{l}f_{D_{S}}= \\
160 \mathrm{Gta}^{-1}\end{array}$ \\
\hline $\begin{array}{l}\varepsilon_{\mathrm{FIX}}= \\
-2 \% 0\end{array}$ & $\begin{array}{l}\varepsilon_{\mathrm{DW}}= \\
-20 \%\end{array}$ & $\begin{array}{l}\varepsilon_{D S}= \\
-2 \%\end{array}$ \\
\hline Marine & \multicolumn{2}{|c|}{$\overbrace{}^{\varepsilon_{\mathrm{p}}}=0 \%\left(\mathrm{NO}^{-}\right)$} \\
\hline Seawater $\delta^{15} \mathrm{~N}_{\mathrm{NO}_{3}^{-}}$ & $\overbrace{\varepsilon_{\mathrm{RE}}}=$ & \\
\hline
\end{tabular}

Fig. C1. Marine nitrogen cycle models. (a) Fully integrated marine-terrestrial-atmosphere N cycle. (b) Simplified N cycle model, which is limited to the most important source flux (cyanobacterial $\mathrm{N}$ fixation) and sink fluxes (sedimentary and water-column denitrification) of seawater fixed N. Fluxes $(f)$ and fractionation factors $(\varepsilon)$ are shown for cyanobacterial fixation (FIX), soil volatilization (VOL), terrestrial denitrification (DT), riverine transport (RIV), atmospheric deposition (DEP), water-column denitrification (DW), sedimentary denitrification (DS), the anammox process (ANX), organic burial (BUR), sedimentary ammonification (AMM), and weathering (W). Sources of model parameters and discussion of model output are given in the text.

were calculated as follows.

$$
\begin{aligned}
& M_{a}(t+1)=M_{a}(t)+\int_{t}^{t+1} \\
& \left(f_{\mathrm{VOL}}+f_{\mathrm{DT}}+f_{\mathrm{DW}}+f_{\mathrm{DS}}+f_{\mathrm{ANX}}\right. \\
& \left.+f_{\mathrm{W}}-f_{\mathrm{FIX}-\mathrm{T}}-f_{\mathrm{DEP}}-f_{\mathrm{FIX}}\right) \\
& M_{t}(t+1)=M_{t}(t)+\int_{t}^{t+1} \\
& \left(f_{\mathrm{FIX}-\mathrm{T}}-f_{\mathrm{VOL}}-f_{\mathrm{DT}}-f_{R I V}\right) \\
& M_{o}(t+1)=M_{o}(t)+\int_{t}^{t+1} \\
& \left(f_{\mathrm{RIV}}+f_{\mathrm{DEP}}+f_{\mathrm{FIX}}-f_{\mathrm{DW}}-f_{\mathrm{DS}}-f_{\mathrm{ANX}}-f_{\mathrm{BUR}}\right) \\
& M_{s}(t+1)=M_{s}(t)+\int_{t}^{t+1}\left(f_{\mathrm{BUR}}-f_{\mathrm{W}}\right)
\end{aligned}
$$

Equations (C2-C5) integrate source and sink fluxes over a specified time range $t$ to $t+1$ for the following processes: ter- restrial nitrogen fixation (FIX-T), volatilization of soil nitrogen (VOL), terrestrial soil denitrification (DT), riverine flux of fixed N (RIV), oceanic nitrogen fixation (FIX), deposition of atmospheric nitrogen in ocean (DEP), water-column denitrification (DW), sedimentary denitrification (DS), anammox loss of fixed N (ANX), burial of organic nitrogen (BUR), and weathering (W) (Fig. C1a). The simplified version of the model is limited to the fluxes FIX, DW, and DS (Fig. C1b). Changes in the $\mathrm{N}$-isotopic composition of a given reservoir $x\left(\delta_{x}\right)$ from $t$ to $t+1$ were calculated as

$$
\begin{aligned}
& \delta_{x}(t+1)=M_{x}(t) \times \delta_{x}(t)+\left(\Sigma_{i=1}^{10}\left(\int_{t}^{t+1} f_{i} \times\left(\delta_{x}+\varepsilon_{i}\right)\right)\right) / \\
& \left(M_{x}(t)+\Sigma_{i=1}^{10}\left(\int_{t}^{t+1} f_{i}\right)\right)
\end{aligned}
$$

where $\varepsilon_{i}$ is the fractionation associated with flux $i$. The equation above is an expression of isotopic mass balance, where the summation ( $\Sigma i=1$ to 10$)$ for each reservoir $x$ was limited to those fluxes $\left(f_{i}\right)$ appearing in Eqs. (C2-C5). 


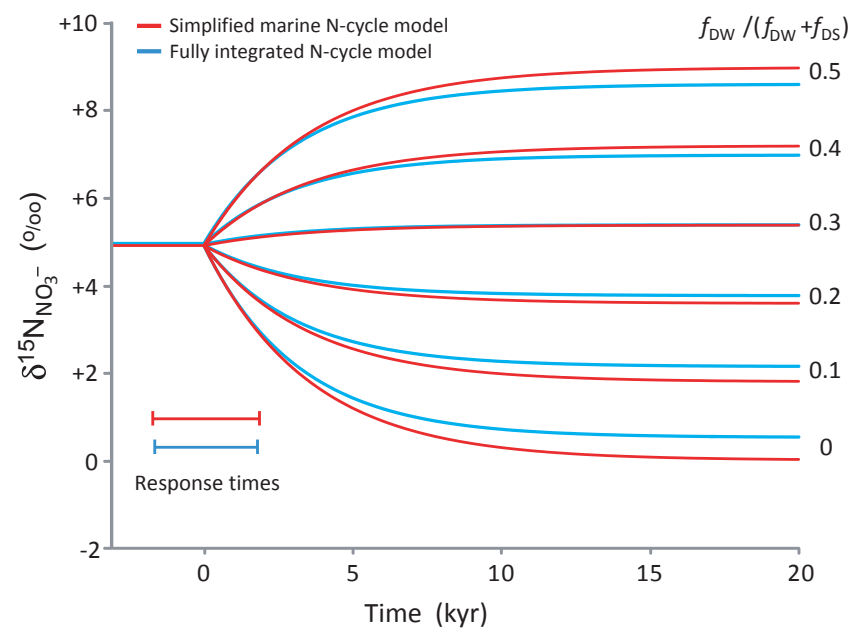

Fig. C2. Comparison of output of fully integrated and simplified $\mathrm{N}$ cycle models. At $t \leq 0$, the model is at equilibrium and represents our "baseline" scenario $\left(f_{\mathrm{FIX}}=220 \mathrm{Tg} \mathrm{a}^{-1}, f_{\mathrm{DW}}=60 \mathrm{Tg} \mathrm{a}^{-1}\right.$, $f_{\mathrm{DS}}=160 \mathrm{Tg} \mathrm{a}^{-1}, \varepsilon_{\mathrm{FIX}}=-2 \%, \varepsilon_{\mathrm{DS}}=-2 \%, \varepsilon_{\mathrm{DW}}=-20 \%$, $\varepsilon_{\mathrm{P}}=0 \%$, and $\left.F_{\mathrm{DW}}=0.27\right)$, which yields a seawater $\delta^{15} \mathrm{~N}_{\mathrm{NO}_{3}^{-}}$ value of $+4.9 \%$ o (i.e., equivalent to modern seawater nitrate; Sigman et al., 2000). At $t=0$, changes in $F_{\mathrm{DW}}$ to values ranging from 0 to 0.5 result in evolution of $\delta^{15} \mathrm{~N}_{\mathrm{NO}_{3}^{-}}$at a rate reflecting the response time of the system (which is closely related to the $\sim 3 \mathrm{kyr}$ residence time of nitrate in seawater; Tyrrell, 1999). Differences in $\delta^{15} \mathrm{~N}_{\mathrm{NO}_{3}^{-}}$between the fully integrated model (blue curves) and simplified model (red curves) are generally $<0.4 \%$, indicating that the output of the simplified model is robust.

We parameterized the model based on the modern marine $\mathrm{N}$ budget. The total mass of seawater nitrate is $\sim 8.0 \times 10^{5} \mathrm{Tg} \mathrm{N}$ (Brandes and Devol, 2002). Whether the present-day marine $\mathrm{N}$ cycle is in balance is a matter of debate (Codispoti, 1995; Brandes and Devol, 2002). Recent studies have documented strong spatial coupling of cyanobacterial $\mathrm{N}$ fixation and water-column denitrification in the modern ocean (Galbraith et al., 2004; Deutsch et al., 2007; Knapp et al., 2008), implying that short-term losses of fixed $\mathrm{N}$ are locally compensated. At longer timescales, losses of fixed $\mathrm{N}$ to denitrification must be balanced by new $\mathrm{N}$ fixation in order to maintain a $\mathrm{N}: \mathrm{P}$ ratio in global seawater close to that of marine phytoplankton (16:1) (Tyrrell, 1999). Estimates of the fluxes associated with cyanobacterial $\mathrm{N}$ fixation $\left(f_{\mathrm{FIX}}\right)$, sedimentary denitrification $\left(f_{\mathrm{DS}}\right)$, and watercolumn denitrification ( $\left.f_{\mathrm{DW}}\right)$ vary widely in older literature, although recent analyses of large data sets are beginning to converge on a consensus range of values (DeVries et al., 2012, 2013; Eugster and Gruber, 2012; Gro $\beta$ kopf et al., 2012). Estimates of $f_{\text {FIX }}$ include $120-140 \mathrm{Tg} \mathrm{a}^{-1}$ (Gruber and Sarmiento, 1997; Galloway et al., 2004; Gruber and Galloway, 2008), 131-134 $\mathrm{Tg} \mathrm{a}^{-1}$ (Eugster and Gruber, 2012), and $177 \pm 8 \mathrm{Tga}^{-1}$ (Gro $\beta$ kopf et al., 2012). Total oceanic denitrification has been estimated at $145-185 \mathrm{Tg} \mathrm{a}^{-1}$
(Gruber and Sarmiento, 1997; Galloway et al., 2004), 120$240 \mathrm{Tg} \mathrm{a}^{-1}$ (DeVries et al., 2013), $230 \pm 60 \mathrm{Tg} \mathrm{a}^{-1}$ (DeVries et al., 2012), 230-285 $\mathrm{Tg} \mathrm{a}^{-1}$ (Middelburg et al., 1996), $240 \mathrm{Tg} \mathrm{a}^{-1}$ (Gruber and Galloway, 2008), and $>400 \mathrm{Tg} \mathrm{a}^{-1}$ (Codispoti, 2007). The relative importance of sedimentary versus water-column denitrification was not well known in the past (Gruber and Sarmiento, 1997), but recent marine N budgets have provided independent estimates of each flux. Estimates for $f_{\text {DW }}$ include $52 \pm 13 \mathrm{Tg} \mathrm{a}^{-1}$ (Eugster and Gruber, 2012) and $66 \pm 6 \mathrm{Tg} \mathrm{a}^{-1}$ (DeVries et al., 2012), while estimates for $f_{\mathrm{DS}}$ range from $93 \pm 25 \mathrm{Tg} \mathrm{a}^{-1}$ (Eugster and Gruber, 2012) to $164 \pm 60 \mathrm{Tg} \mathrm{a}^{-1}$ (DeVries et al., 2012) and $>300 \mathrm{Tg} \mathrm{a}^{-1}$ (Codispoti, 2007).

The $\delta^{15} \mathrm{~N}$ of seawater nitrate in the deep ocean (i.e., the largest reservoir of fixed $\mathrm{N}$ ) is +4.8 to $+5.0 \%$ (Sigman et al., 2000). The $\delta^{15} \mathrm{~N}$ of present-day atmospheric $\mathrm{N}_{2}$ is $0 \%$ o (Mariotti, 1984), a value inferred to have been nearly invariant through time (Berner, 2006b). The fractionation associated with cyanobacterial $\mathrm{N}$ fixation of atmospheric $\mathrm{N}_{2}\left(\varepsilon_{\mathrm{FIX}}\right)$ is estimated to be -1 to $-3 \%$ (Macko et al., 1987; Carpenter et al., 1997). Fractionation during water-column denitrification $\left(\varepsilon_{\text {DW }}\right)$ has a maximum value of $\sim-27( \pm 3) \%$ (Gruber and Sarmiento, 1997; Barford et al., 1999; Voss et al., 2001; Murray et al., 2005), although the effective fractionation may be closer to $-20 \%$ (Brandes and Devol, 2002). Recent culture studies have suggested that this fractionation might even be as low as -10 to $-15 \%$ (Kritee et al., 2012), an idea that we explore in our modeling simulations. We did not parameterize the anammox reaction (Sigman et al., 2003) separately owing to significant uncertainties concerning the scale of this process and any associated fractionation. While this reaction is a major sink for seawater fixed $\mathrm{N}$, possibly larger than water-column denitrification in some oceanic regions (Mulder et al., 1995; Kuypers et al., 2005), it is thought that field-based estimates of fractionation due to water-column denitrification have incorporated any effects related to anammox (Thamdrup et al., 2006). Denitrification in suboxic marine sediments $\left(\varepsilon_{\mathrm{DS}}\right)$ typically yields a small net fractionation $(\sim-1$ to $-3 \%$ ) owing to near-quantitative utilization of porewater nitrate (Lehmann et al., 2004; Galbraith et al., 2008). However, this fractionation can range from $\sim 0 \%$ in organic-rich, reactive sediments to as high as -5 to $-7 \%$ in organic-lean, unreactive sediments (Lehmann et al., 2007). An estimate of $-0.8 \%$ o for the global mean fractionation due to sedimentary denitrification (Kuypers et al., 2005) does not take into account effects associated with the upward diffusive flux of ${ }^{15} \mathrm{~N}$-enriched ammonium in reactive sediments (Higgins et al., 2012). The fractionation associated with assimilation of seawater fixed $\mathrm{N}$ by eukaryotic marine algal $\left(\varepsilon_{\mathrm{P}}\right)$ can be as large as -5 to $-8 \%$ o for nitrate (Lehmann et al., 2007) but is more typically -1 to $-3 \%$ (Macko et al., 1987; Carpenter et al., 1997). We assumed a net fractionation of $0 \%$ based on complete photosynthetic utilization of seawater nitrate at longer timescales. The fractionation associated with ammonium uptake by marine algae is $-10( \pm 5) \%$ (Brandes 
and Devol, 2002), and this process is more likely to yield a net fractionation owing to incomplete utilization of recycled ammonium in anoxic marine systems (Higgins et al., 2012).

The fully integrated and simplified marine N-cycle models exhibit similar results for seawater $\delta^{15} \mathrm{~N}_{\mathrm{NO}_{3}^{-}}$under a wide range of conditions. The models were tuned to yield identical $F_{\mathrm{DW}}$ of 0.27 for the baseline scenario, in which seawater $\delta^{15} \mathrm{~N}_{\mathrm{NO}_{3}^{-}}$is equal to $+4.9 \%$ (Fig. C2). Varying $F_{\mathrm{DW}}$ from 0 to 0.5 yields a slightly smaller range of $\delta^{15} \mathrm{~N}_{\mathrm{NO}_{3}^{-}}$values for the fully integrated model (from $+0.53 \%$ o to $+8.60 \%$ ) than for the simplified model (from $+0 \%$ to $+9.0 \%$ ), indicating that inclusion of secondary fluxes in the fully integrated model has a modest stabilizing effect on the isotopic composition of seawater nitrate.
Differences in $\delta^{15} \mathrm{~N}_{\mathrm{NO}_{3}^{-}}$between the two models are $<0.4 \%$ o for the range of $F_{\mathrm{DW}}(\sim 0.1-0.5)$ that is most likely to characterize natural systems (Fig. C2). This outcome reflects the fact that the secondary fluxes present in the fully integrated model (e.g., atmospheric deposition, riverine transport, and burial; Fig. C1a) are generally either small in magnitude or associated with limited fractionations. Thus, the simplified model (Fig. C1b) provides a reasonably robust representation of the major influences on the $\mathrm{N}$-isotopic composition of seawater nitrate. 